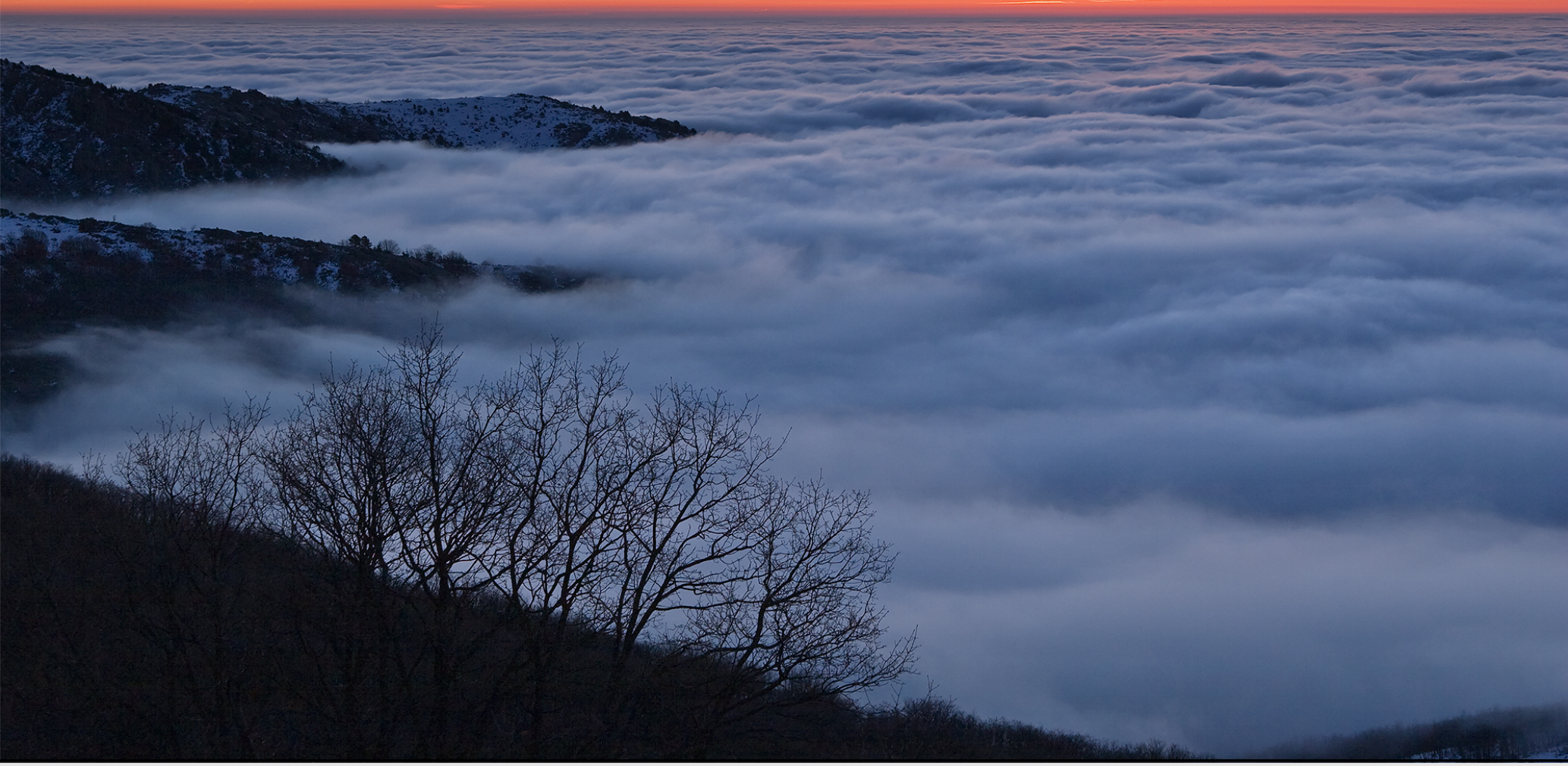

\title{
Diseño y construcción de SPC: error en el modelo
}

\author{
Alfons Callado i Pallarés \\ Delegación territorial (DT) en Cataluña, Agencia Estatal de Meteorología (AEMET)
}

\begin{abstract}
I believe that the ultimate climate models ... will be stochastic, i.e. random numbers will appear somewhere in the time derivatives.

Creo que en última instancia los modelos climáticos ... serán estocásticos, es decir, aparecerán números aleatorios en algún lugar de las derivadas temporales.
\end{abstract}

EDWARD NORTON LORENZ, 1969

Las ecuaciones con las que describimos la evolución futura de la atmósfera son de naturaleza caótica, muy sensibles a pequeñas variaciones en las condiciones iniciales, de modo que las soluciones de simular la atmósfera no son convergentes y debemos tener en cuenta las incertidumbres en las condiciones iniciales. Pero, además, los modelos de predicción del tiempo no son perfectos, son sólo buenas aproximaciones para describir la evolución de la atmósfera, con un conjunto importante de limitaciones que hace que lleven asociados errores e incertidumbres. Así, se nos plantean dos grandes retos: por un lado, tener en cuenta las incertidumbres en las condiciones iniciales y, por otro lado, tener en cuenta los errores, limitaciones e incertidumbres inherentes en los modelos. Con un solo modelo determinista no podemos plantearnos estos retos, pero sí con los sistemas de predicción por conjuntos (SPC). Abordamos, en este capítulo, el segundo reto: los errores, limitaciones e incertidumbres inherentes en los modelos.

Palabras clave: diseño de sistemas de predicción por conjuntos, incertidumbres en los modelos, método multimodelo, método multianálisis, método multicontorno, método multifísica, método multiparámetro, parametrizaciones estocásticas. 


\subsection{Introducción}

Vimos en el capítulo 5 en la página 49 que las ecuaciones en los modelos de predicción del tiempo que describen la evolución futura de la atmósfera tienen un comportamiento caótico y que son muy sensibles a pequeñas variaciones en las condiciones iniciales: las soluciones de simular la atmósfera no son convergentes. Por ejemplo y, exagerando un poco, simulando el tiempo para la península ibérica para dos días tomando la observación de una temperatura de $-3.4^{\circ} \mathrm{C}$ en Reykiavik (Islandia) nos podría dar «sol y moscas» (tiempo estable o buen tiempo), mientras que asimilando una observación de $-3.36{ }^{\circ} \mathrm{C}$, es decir, dentro del margen de error en la observación, nos podría dar chubascos o mal tiempo: dos predicciones totalmente diferentes.

Pero no es el único reto de los SPC tener en cuenta estas incertidumbres en las condiciones iniciales tal como se ha descrito en el capítulo 16 en la página 243. Los modelos de predicción del tiempo no son perfectos. En realidad, los modelos son sólo buenas aproximaciones para describir la evolución de la atmósfera. Son el fruto de los conocimientos y experiencia humanos en física, matemáticas, meteorología, tecnología, etc. que se traducen en unos programas informáticos que simulan la evolución de la atmósfera y que se ejecutarán en un superordenador (cap. 11 en la página 145). Así, debido a la inclusión de multitud de aproximaciones para su resolución, las limitaciones del conocimiento en ciertos procesos atmosféricos y la intrínseca naturaleza caótica de las ecuaciones físicas que describen la atmósfera, así como la limitación física en el cálculo de los superordenadores, hace que los modelos sean inherentemente no perfectos, con errores e incertidumbres.

En consecuencia, tener en cuenta los errores, limitaciones y las incertidumbres inherentes en los modelos es el otro gran reto de los SPC, además del ya discutido de las incertidumbres en las condiciones iniciales (cap. 16 en la página 243). Cabe resaltar que las implicaciones para un SPC, en ambos casos, son bastante parecidas.

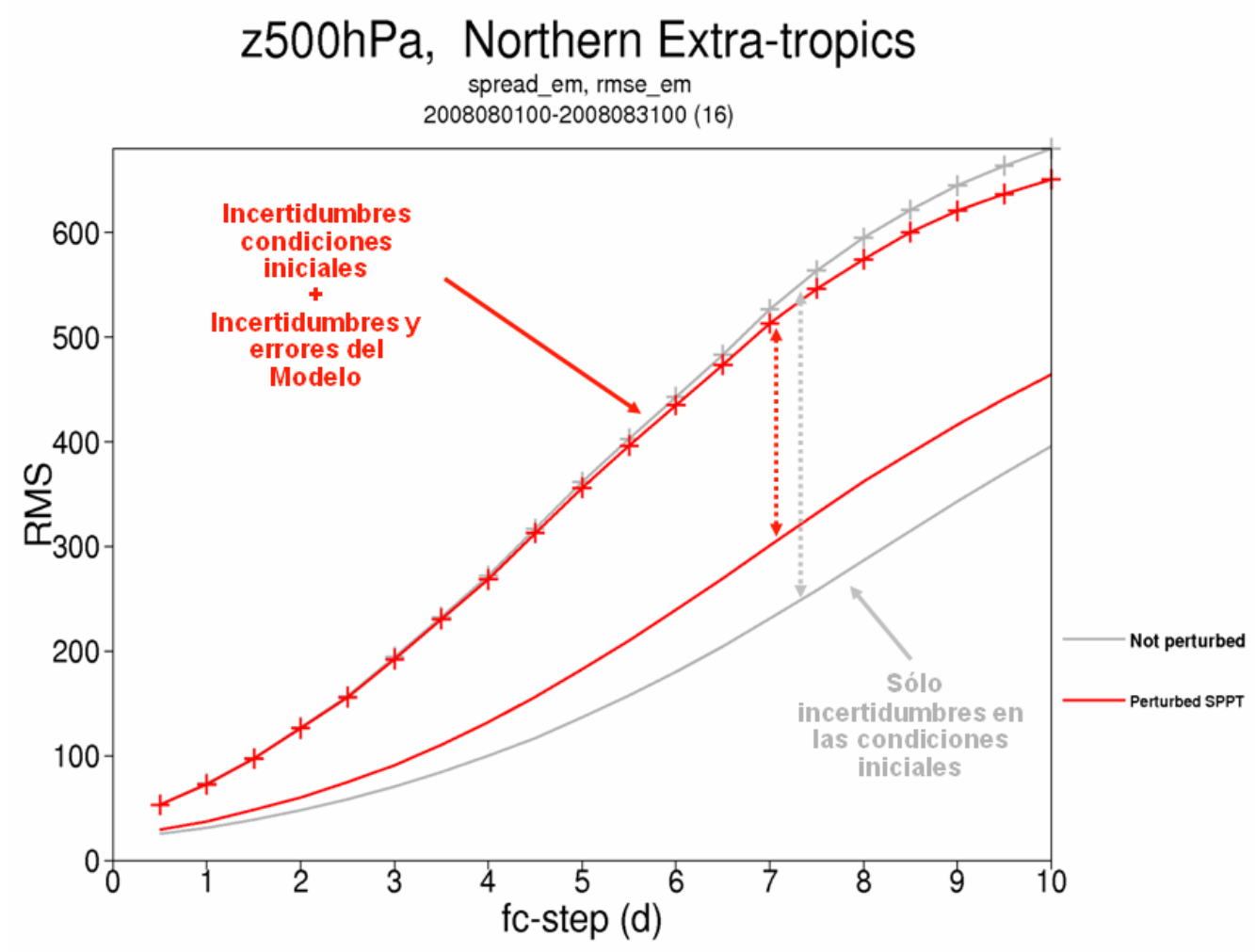

Figura 17.1: Diagrama de evolución con el alcance predictivo de dispersión y error de la altura geopotencial en $500 \mathrm{hPa}$, para el SPC del ECMWF ECENS teniendo en cuenta: en las líneas grises sólo la incertidumbre en las condiciones iniciales y, en las rojas, la de las condiciones iniciales más las del propio modelo. En las respectivas líneas verticales discontinuas con flechas se representa la distancia entre las curvas de dispersión (continuas sólidas) y error (continuas con cruces). 


\subsection{Historia y conceptos básicos}

Históricamente fue a finales del siglo XX cuando los principales centros meteorológicos a nivel mundial que utilizaban SPC globales se plantearon y empezaron a aplicar las primeras técnicas para tener en cuenta en sus SPC los errores e incertidumbres de los modelos. Más tarde, desde los albores del siglo XXI estos conceptos y técnicas se han empezado también a aplicar a los SPC regionales.

Surgió casi por una necesidad, a un nivel más práctico que teórico: los SPC adolecían de falta de dispersión (sec. 13.6.5 en la página 181) y, por ende, de consistencia y fiabilidad (sec. 15.8 en la página 222), sobre todo en alcances predictivos grandes tales como los 3-7 días, lo que se denomina medio plazo (cap. 4 en la página 29).

Desde un punto de vista práctico, lo podemos ver en la Figura 17.1 en la página anterior para el ECENS (SPC global del ECMWF con el modelo IFS, sec. 19.3 en la página 293): sin tener en cuenta los errores del modelo las curvas de dispersión (línea gris continua) y error (línea gris con cruces) están cada vez más lejos, cuando para un SPC consistente las dos deberían aumentar al mismo ritmo: es decir, cuando más cercanas mejor (sec. 15.8.2 en la página 223).

Si se aplica la técnica denominada, de momento y abreviadamente, SPPT (se describirá más adelante en el presente capítulo) para tener en cuenta errores/incertidumbres del modelo, las cosas mejoran, ambas curvas se aproximan, con un aumento notable de la dispersión (línea roja continua) e incluso con disminución del error al subir la curva correspondiente (línea roja con cruces) un poco más lentamente.

Las mejorías de los SPC globales como las mostradas en la Figura 17.1 en la página anterior justificaron a nivel práctico tener en cuenta errores e incertidumbres de los modelos en los SPC.

A nivel teórico, en un SPC y, a pesar de tener en cuenta las incertidumbres en las condiciones iniciales, si todos sus miembros utilizan exactamente el mismo modelo con las mismas configuraciones, éste tiene su propia climatología que lo limita para explorar otros espacios de fases (digamos otras atmósferas posibles u otras posibilidades de predicción) que son totalmente plausibles. Podemos verlo en la Figura 17.2.

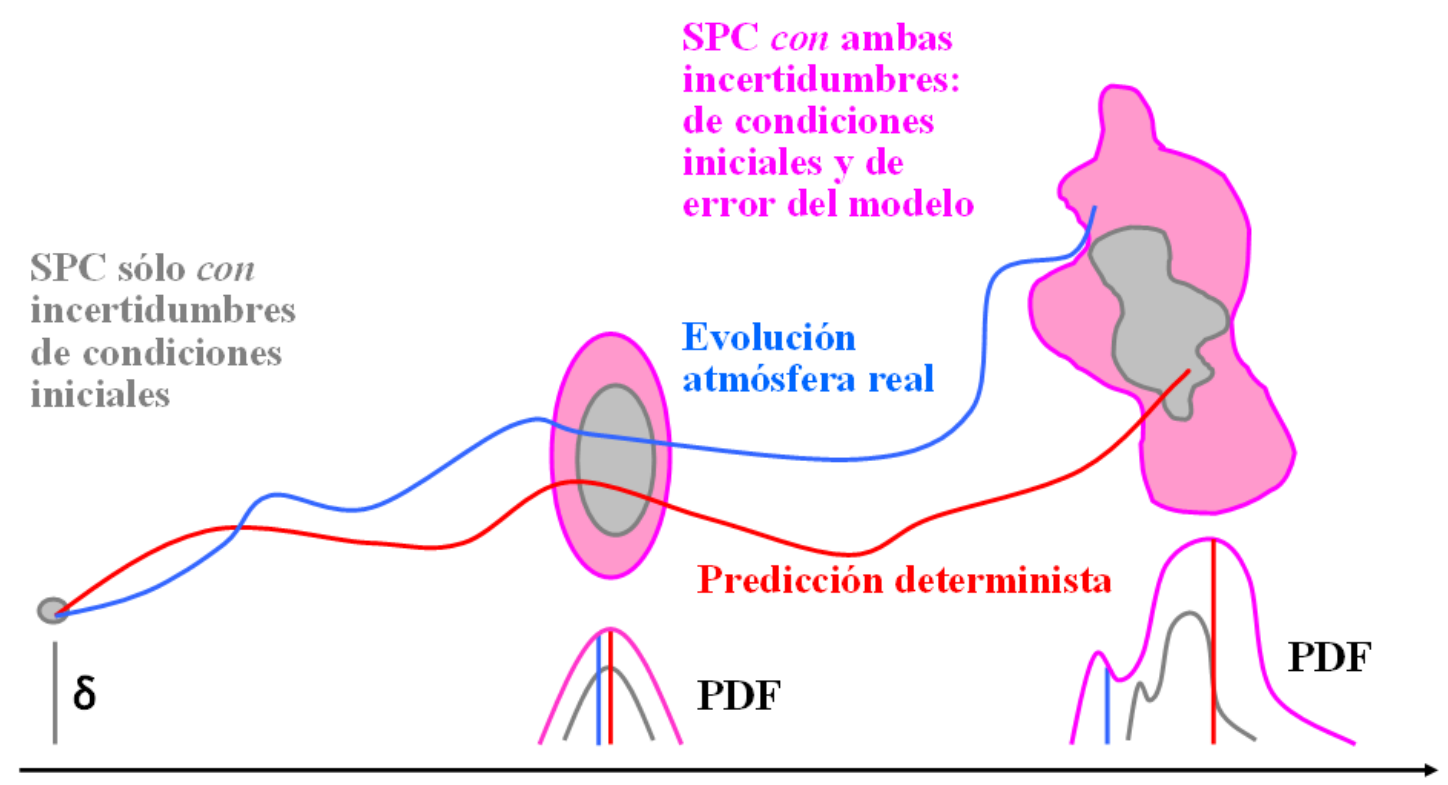

Alcance de Predicción

Figura 17.2: Esquema de la predicción de la evolución de la atmósfera con dos SPC: en gris, uno que sólo tiene en cuenta la incertidumbre en las condiciones iniciales y, en magenta, otro que además tiene en cuenta las incertidumbres o errores del modelo. En rojo se representa una predicción con un solo modelo o predicción determinista y, en azul, la evolución real observada a posteriori de la atmósfera. 


\begin{tabular}{|c|c|c|}
\hline $\begin{array}{l}\text { Clasificación de errores e } \\
\text { incertidumbres }\end{array}$ & Características / Tipos & Métodos en SPC \\
\hline errores sistemáticos & $\begin{array}{c}\text { Oestes demasiado intensos } \\
\text { QBO } \\
\text { MJO } \\
\text { Etc. }\end{array}$ & - \\
\hline \multirow[t]{2}{*}{ errores/incertidumbres aleatorias } & $\begin{array}{l}\text { Fluctuaciones estadísticas en la } \\
\text { Física }\end{array}$ & $\begin{array}{c}\text { Parametrización estocástica } \\
\text { SPPT } \\
\text { MultiFísica } \\
\end{array}$ \\
\hline & $\begin{array}{c}\text { Transferencia energía cinética a } \\
\text { mayores escalas } \\
\text { Etc... }\end{array}$ & $\begin{array}{c}\text { Parameterización estocástica } \\
\text { SKEB }\end{array}$ \\
\hline incertidumbres conocidas & $\begin{array}{c}\text { Valores de parámetros en la física } \\
\text { (e. g. límite de inestabilidad para } \\
\text { disparo de la convección } \\
\text { parametrizada) }\end{array}$ & Multiparámetros \\
\hline incertidumbres desconocidas & - & $\begin{array}{l}\text { ¿Multimodelo? } \\
\text { «La caja negra» }\end{array}$ \\
\hline
\end{tabular}

Tabla 17.1: Tentativa de clasificación de errores e incertidumbres de los modelos, inspirada en las lecciones del experto GLENN SHUTTS y que incluye en la última columna los métodos aplicados en los SPC para tenerlos en cuenta.

En la Figura 17.2 en la página anterior podemos apreciar que un SPC que tiene en cuenta sólo las incertidumbres en las condiciones iniciales, explora (mancha gris) unas posibles evoluciones de la atmósfera (regiones del espacio de fases).

La mancha gris crece con el alcance de la predicción al aumentar la incertidumbre, es decir, disminuye la predecibilidad. Cuanto más extensa es la mancha, menor es la predecibilidad: menos concreta podrá ser una predicción del tiempo debido a la mayor incertidumbre.

Hemos representado la evolución real de la atmósfera con una línea azul y, en el último alcance, la evolución real de la atmósfera está fuera de nuestra mancha azul: malas noticias. La evolución de la atmósfera no está dentro de nuestra mancha gris, es decir, no es una evolución considerada por nuestro SPC.

$\mathrm{Y}$ ahora viene la magia. Si tenemos en cuenta en el SPC errores e incertidumbres inherentes de nuestro modelo, simulamos la evolución de la mancha magenta que crece más, explorando más posibilidades (más regiones del espacio de fases con más grados de libertad) y que incluye, en el último alcance, la evolución de la atmósfera real: ahora nuestro SPC es consistente y fiable, describiendo y acotando adecuadamente la predecibilidad.

\subsection{Errores e incertidumbres del modelo}

La Tabla 17.1, inspirada en una de las lecciones de uno de los expertos en el error del modelo, GLENN SHUTTS [22], intenta clasificar, basándose en los conocimientos científicos, lo que los meteorólogosmodelizadores piensan y creen que son los errores e incertidumbres en los modelos.

Diferentes aspectos de la Tabla 17.1 serán tratados a lo largo del capítulo. Destacamos aquí sólo uno: la distinción entre errores e incertidumbres, una distinción que en algunos capítulos del libro no se tiene en cuenta por no llegar a ese nivel de detalle.

\subsubsection{Errores versus incertidumbres}

No hay unanimidad en la comunidad científica en meteorología en una distinción conceptual clara de a qué nos referimos exactamente cuando hablamos de errores o a qué cuando hablamos de incertidumbres en los modelos. Aquí, para el presente capítulo, optamos por la siguiente: los errores del modelo son aquellas deficiencias intrínsecas en el mismo que lo incapacitan para simular sistemáticamente de forma adecuada evoluciones atmosféricas observadas. 


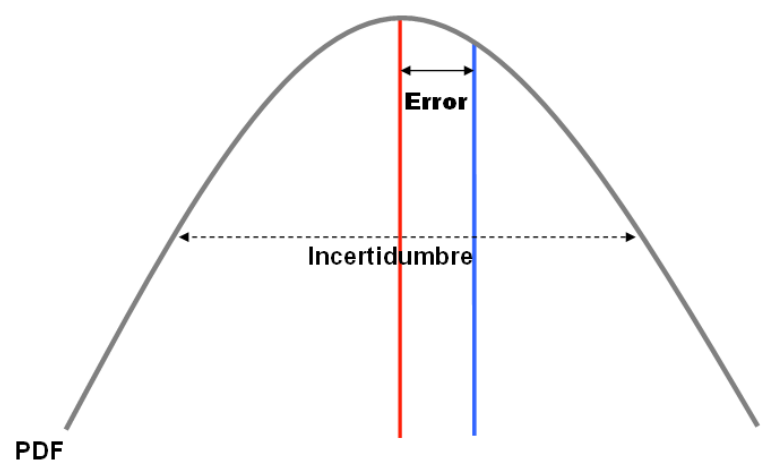

Figura 17.3: Esquema de la diferencia conceptual entre error e incertidumbre del modelo en un evento atmosférico. En azul el evento observado en la atmósfera real. En rojo una predicción determinista. En la campana $o$ PDF en gris, las posibles predicciones del modelo con pequeños cambios: la predicción en rojo es la media.

Cualesquiera que sean las mejoras introducidas en un modelo, éstas tienden a hacer los errores más pequeños e incluso eventualmente solucionarlos. Por ejemplo, imaginemos un modelo que tiene dificultades en simular tormentas de evolución diurna de montaña que descargan por las tardes en situaciones anticiclónicas de buen tiempo. Concretando, el modelo predice siempre en estas situaciones chubascos moderados cuando en realidad hay tormentas fuertes con granizo: infravalora la convección sistemáticamente. Esto es un error del modelo susceptible de ser mejorado.

Y para las incertidumbres adoptamos la siguiente definición: las incertidumbres del modelo serán aquellas limitaciones en la descripción física de procesos atmosféricos, bien por incertidumbre inherente o bien por la limitación de conocimientos científicos codificados en los modelos, que a su vez limitan la simulación precisa de las evoluciones atmosféricas observadas. Concretemos con un ejemplo similar al anterior. Ahora nuestro modelo sí que simula las tormentas de tarde, pero debido a la baja predecibilidad del mismo fenómeno atmosférico y las limitaciones en cómo se simulan las tormentas en nuestro modelo, a veces las tormentas simuladas son más fuertes que las observadas, a veces más bien moderadas o incluso débiles, a veces hay más tormentas que las observadas, a veces hay menos, etcétera. Y ya lo tenemos, nuestro modelo es capaz de simular las tormentas fuertes con granizo, pero está limitado en simularlas de manera precisa y exacta, de modo que alrededor de lo que es capaz de simular estará la solución exacta. Es decir, hay incertidumbre en nuestro modelo.
En la Figura 17.3 se han esquematizado los dos conceptos de error e incertidumbre. El error es la diferencia sistemática de la media de todas las predicciones de un evento atmosférico respecto a la observación de éste. La incertidumbre es la variedad de predicciones que se pueden tener alrededor del valor bueno, debido a pequeñas variaciones, por ejemplo, en las condiciones iniciales. Y, para finalizar, como se representa en la figura, debe señalarse que se pueden tener ambos a la vez, errores e incertidumbres del modelo. Es más, si la predicción media (línea roja vertical) fuera igual a la observación (línea azul vertical), no tendríamos error pero sí incertidumbre.

\subsubsection{Incertidumbres en la dinámica y en la física}

Clásicamente se ha considerado que los errores e incertidumbres de los modelos están en la física o parametrizaciones físicas o procesos submalla (sec. 10.5 en la página 137) y no en la dinámica o procesos de malla (sec. 10.4 en la página 135). En general, la parte dinámica del modelo se considera muy precisa, mientras que la parte física se considera que puede ser significativamente imprecisa e incluso con una incertidumbre inherente tal como las incertidumbres aleatorias y las conocidas referenciadas en la Tabla 17.1 en la página anterior.

Incertidumbre en la dinámica. Tal y como ya se ha contado, clásicamente se ha considerado que la dinámica albergaba muy poco margen para errores e incertidumbres debido a que se basa en una aplicación directa de las consideradas bien conocidas y fundamentadas leyes de la física clásica de Is A AC NEWTON y otros. Estas leyes se basan en los principios de conservación de la cantidad de movimiento (o momento lineal), la masa y la energía, complementadas con la ley de la gravitación universal y la ley de los gases ideales, además de tener en cuenta la rotación terrestre (la fuerza aparente de Coriolis). Pero siendo concienzudos, se debe admitir que su aplicación práctica en un modelo numérico de predicción del tiempo comporta un buen sinnúmero de aproximaciones, como por ejemplo:

- Aproximaciones en las mismas ecuaciones: tierra esférica, gravedad constante en toda la tierra y en la vertical, etc.

- Aproximaciones diferentes con el aspecto matemático de los métodos numéricos utilizados para 
obtener un conjunto de ecuaciones resoluble que se puedan codificar en unos programas informático.

- La arbitrariedad en la elección de grids o mallas horizontales de ARAKAWA (https://en. wikipedia.org/wiki/Arakawa_grids) y de la elección de distintas discretizaciones de los niveles verticales (eta, sigma híbridos sigmapresión, etc. (http://glossary . ametsoc.org/ wiki/Vertical_coordinate_system) y su ulterior distribución.

- Artificiosos filtros y difusiones (y su tuneo) para que el modelo no se inestabilice y falle, por ejemplo, filtrando las ondas sonoras

- Cómo se incluye la humedad, componente variable de la composición atmosférica

- El acoplamiento entre dinámica y física en los puntos de malla

- Etcétera.

Se ha empezado, pues, en la comunidad de desarrolladores de modelos numéricos de predicción del tiempo a estudiar esta incertidumbre en la dinámica, como por ejemplo en el Dynamical Core Model Intercomparison Project (DCMIP [10, 23], https://earthsystemcog.org/projects/ dcmip-2016/), e incluso a empezar a tenerla en cuenta explícitamente, como por ejemplo en el ECENS [QJ:QJ3094, 13]. Así que en los próximos años y décadas se desarrollarán nuevas generaciones de modelos y de SPC donde se considerarán las incertidumbres en la dinámica explícitamente.

Y no albergamos ninguna duda de que, inevitablemente, en el caso de los SPC, se cumplirá la cita que abre este capítulo de nuestro admirado LORENZ: «Creo que en última instancia los modelos (climáticos) ... serán estocásticos, es decir, aparecerán números aleatorios en algún lugar de las derivadas temporales».

No podemos acabar este apartado sin mencionar que los desarrolladores de sistema(s) de predicción por conjuntos (SPC) multimodelo [11] (ver siguiente apartado), consideran que uno de los puntos fuertes del método es que ya tiene implícitamente en cuenta las incertidumbres en la parte dinámica de sus SPC [18] (sec. 13.5 en la página 174).

Incertidumbre en la física. En estos contextos, la física es incierta por construcción. Las Parametrizaciones físicas se basan en la hipótesis de que los procesos submalla parametrizados están en equilibrio secular con los procesos resueltos en la malla por la dinámica
[17] (sec. 10.4 en la página 135). Expliquemos este equilibrio. Los procesos de la física son descritos aproximadamente a través de lo que ya sabemos de los procesos en la dinámica. Aproximadamente, porque en realidad estamos escogiendo la media de las posibles salidas de la física o procesos submalla de todos aquellos compatibles con la dinámica o procesos de malla.

Física versus parametrizaciones. La física de un modelo engloba a un conjunto de diversas parametrizaciones, tales como parametrización de la convección, de la Planetary Boundary Layer (PBL) o parametrización de la turbulencia, parametrización de las nubes o microfísica de nubes, parametrización de las radiaciones solar y terrestre, etc.

Parametrizar. Describir aproximadamente procesos complejos descritos por muchas variables a través de unas pocas variables bien conocidas. En los modelos de predicción del tiempo, significa inferir la influencia de los procesos submalla con las variables bien conocidas de la malla. Cabe resaltar que, dado que los procesos de la atmósfera en todas sus escalas forman un continuo interaccionando entre todas ellas, no hay una justificación teórica para truncar y separar su descripción en procesos de malla y submalla. Parametrizar es un razonable invento práctico que hace posibles las predicciones con modelos numéricos del tiempo sin tener que llegar a la escala molecular, que imposibilitaría hacer predicciones del tiempo con la capacidad de cálculo con los superordenadores actuales o futuros. Se han propuesto otras maneras de parametrizar, por ejemplo, no hacerlo sólo en un punto de malla, sino en varios [17].

El límite de las parametrizaciones. Parametrizar era un buen recurso cuando los modelos tenían una resolución horizontal de unos $100 \mathrm{~km}$ y tenía sentido hacerlo porque se podía sostener la hipótesis de que los procesos submalla estaban en equilibrio secular con los procesos de malla. Al aumentar la resolución de los modelos, tal hipótesis se resquebraja: ahora hay una multitud de procesos submalla compatibles con un proceso de malla. Crece la incertidumbre de los procesos submalla con la resolución horizontal de los modelos. ¿Pero por qué se siguen utilizando las parametrizaciones en los modelos? Pues debido a que la incertidumbre y/o errores introducidos por la física son compensados, y con creces, por la mejoría de la dinámica simulada a escalas más pequeñas [21]. 

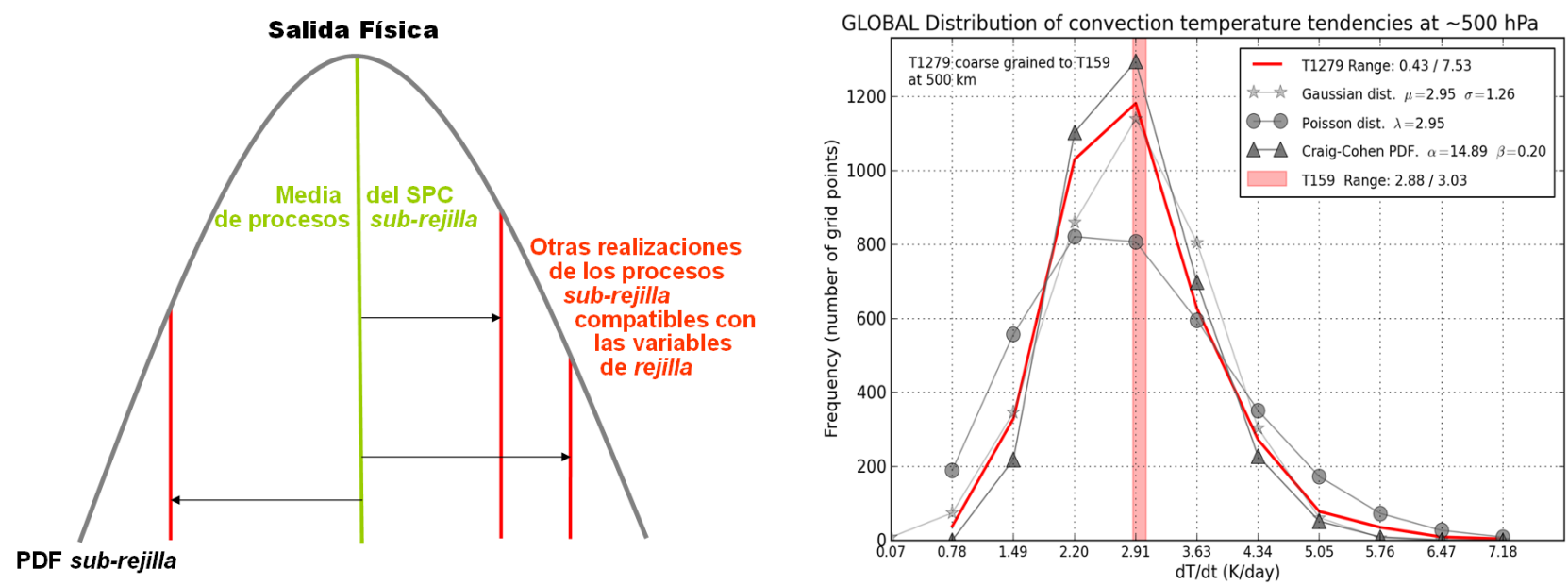

Figura 17.4: Incertidumbre en la física: teoría (izquierda) y práctica (derecha). En la teoría (izquierda), la incertidumbre inherente de la física o las fluctuaciones estadísticas, las conocidas de la Tabla 17.1 en la página 260, son las diversas salidas de la física o procesos submalla (curva PDF submalla en gris) compatibles con las variables de malla, ejemplificadas en tres realizaciones (líneas rojas). Como proceso submalla en un modelo se toma la media (línea verde). En la práctica (derecha), se muestra la distribución de las posibles salidas de un proceso submalla, salidas de la tendencia de la temperatura en la parametrización de la convección (en rojo), de la que se toma en la malla la media representada por la barra vertical (rosa). Se muestran también posibles ajustes de curvas a la distribución (curvas grises con símbolos): gaussiano (estrellas), Poisson (círculos) y Craig-Cohen (triángulos).

Podemos ver este proceso en la Figura 17.4 izquierda, donde las parametrizaciones escogen el valor medio, pero para la atmósfera real otros valores son compatibles y posibles en la submalla, sin poder saber de antemano cual es el valor correcto. Es lo que en la Tabla 17.1 en la página 260 se referencia como fluctuaciones estadísticas de la física en incertidumbres aleatorias. Se puede comprobar, en la práctica, cuando utilizando la técnica coarse training (ver más adelante), se plotea la distribución (campana roja) real del proceso de submalla de la parametrización de la convección alrededor del valor medio (barra vertical rosa) tomado por la malla del modelo. Por otro lado y, para echar más leña al fuego con las incertidumbres en la física, diremos que en ella se utilizan números mágicos, llamados parámetros, que deciden en que dirección va a ocurrir un proceso submalla: por ejemplo, si va a haber o no convección en un punto de malla. Estos números mágicos se infieren o aproximan, normalmente, de manera indirecta, a través de observaciones meteorológicas que no nos dan directamente su valor. Y los hemos llamado mágicos porque realmente no conocemos su valor preciso, sino uno aproximado razonable. Es decir, tenemos otra incertidumbre en la física del modelo en esos parámetros: lo que en la Tabla 17.1 en la página 260 aparece como valores de parámetros en la física en incertidumbres conocidas.
Diagnosticando las incertidumbres. Hay diferentes maneras de diagnosticar y/o evaluar las incertidumbres y/o errores en los modelos de predicción del tiempo. Nosotros vamos a mostrar sólo los resultados del método denominado coarse graining de GLENN ShutTs [21], que se basa en comparar las salidas de un modelo numérico del tiempo operativo como el ECHRES (e. g. versión antigua T159 con $125 \mathrm{~km}$ de resolución) con uno de muy alta resolución (e. g. versión penúltima T1279 con 16 km) que juega el papel de atmósfera real. En la Figura 17.5 en la página siguiente mostramos los resultados para la dinámica, la física y cada proceso de la física. Cuanto más planas son las líneas, menos incertidumbre hay y, cuanto más cerradas son las curvas en forma de $V$, más incertidumbre tenemos. Así que podemos diagnosticar ideas que ya conocíamos y añadir algunas nuevas:

- La física (curva negra) es más incierta que la dinámica (curva magenta), al ser la curva de la física más en forma de $V$ cerrada y la curva de la dinámica más plana.

- La microfísica de nubes (curva verde) o la descripción de las nubes es la parametrización más incierta de todas: la $V$ más cerrada, incluso más que la convección (curva roja) o nubes de tormenta.

- La parametrización de la radiación (curva azul) es la menos incierta, aunque tiene un mínimo hacia los cielos despejados, donde la tierra pierde hacia el espacio energía infrarroja a razón de -1 K / día. 


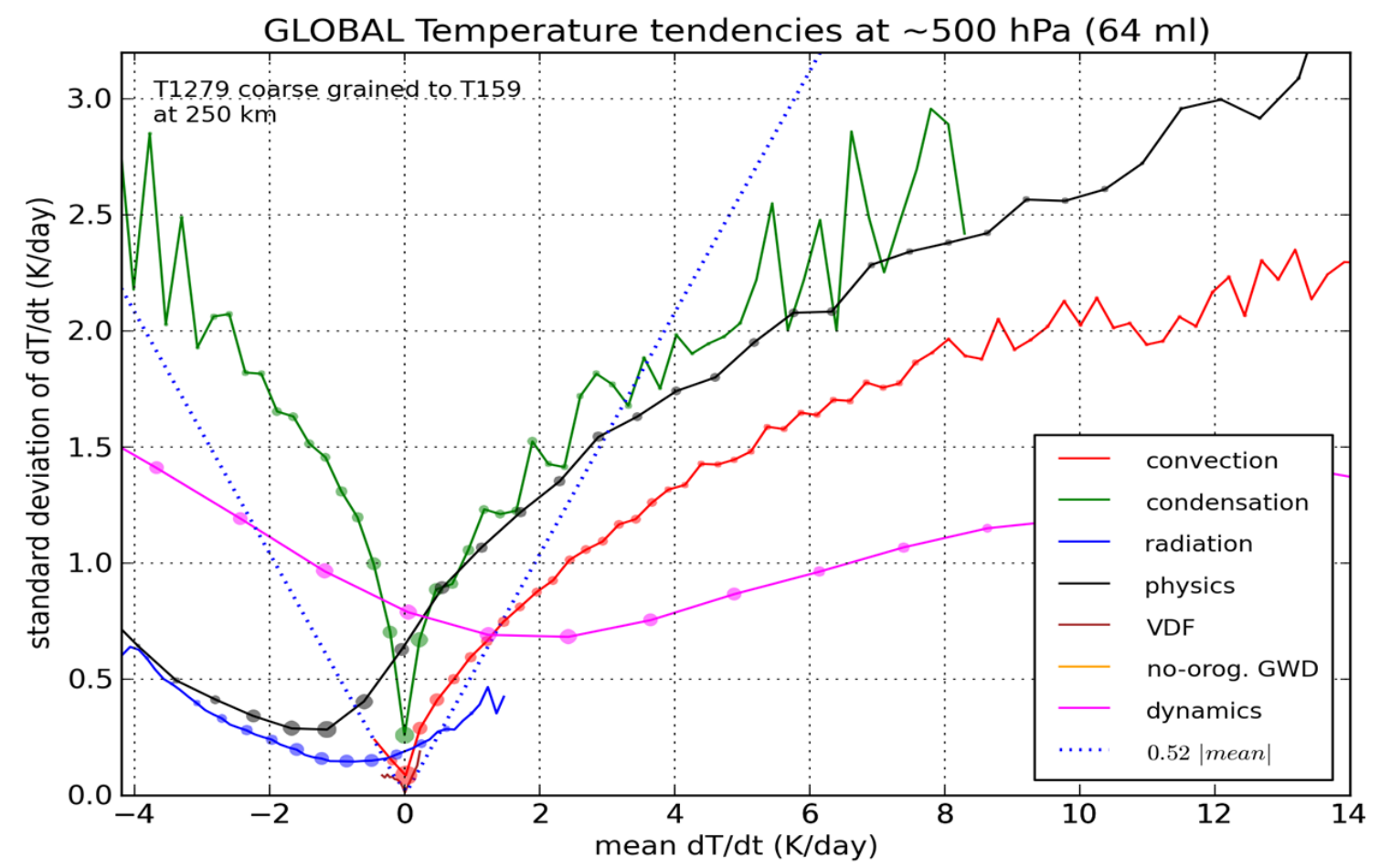

Figura 17.5: Incertidumbres en $500 \mathrm{hPa}$ de la tendencia de la temperatura de la dinámica y la física del ECHRES a $125 \mathrm{~km}$ (T159) de resolución horizontal comparado con la técnica coarse-graining con la supuesta verdad o atmósfera real, representada por el mismo modelo a $16 \mathrm{~km}$ (T1279) de resolución horizontal. Principales incertidumbres representadas: dinámica (magenta), física o parametrizaciones en conjunto (negro) y parametrizaciones individuales de la microfísica de nubes (verde), de la convección (rojo) y de la radiación (azul). Las dos líneas discontinuas azules en forma de V simétrica representan la perturbación de toda la física por la parameterización estocástica SPPT (sec. 17.4.4 en la página 269).

\subsection{Métodos en los SPC para te- ner en cuenta errores e incer- tidumbres en los modelos}

Se utilizan diversas técnicas para tener en cuenta errores e incertidumbres en los SPC. La mayoría de ellas son compatibles entre ellas y potencialmente se pueden aplicar de manera combinada. Las principales técnicas para tener en cuenta las incertidumbres son, sin pretender ser exhaustivos pero sí ilustrativos:

Multiparámetros, que utilizan en diferentes miembros del SPC diferentes valores de parámetros en la física (aquellos números mágicos descritos anteriormente), todos ellos plausibles.

Multifísica, que usa diferentes físicas o diferentes combinaciones de parametrizaciones de la física en diferentes miembros.

Multimodelo, que se fundamenta en utilizar un modelo de predicción del tiempo diferente en cada miembro.
Física, parametrizaciones estocásticas, que se basan en perturbar aleatoriamente toda la física o parte de ella (algunas parametrizaciones) dentro de unos márgenes plausibles de manera diferente en cada miembro del SPC.

Veamos con un poco de detalle cada una de ellas con ejemplos.

\subsubsection{SPC multiparámetros}

Los SPC multiparámetros se basan en utilizar diferentes configuraciones de parámetros dentro de los procesos submalla o parametrizaciones de la física. Ya se ha contado en la incertidumbre de la física que esos parámetros son figurativamente unos números mágicos, que controlan procesos clave de las parametrizaciones, que su valor preciso se desconoce y hay un abanico de posibles valores razonables inferidos desde las observaciones disponibles. 


\begin{tabular}{|c|c|c|c|}
\hline Parametrización & $\begin{array}{l}\text { SPC multiparámetros } \\
\text { Parámetros a perturbar }\end{array}$ & $\begin{array}{l}\text { Valor Medio (por } \\
\text { defecto) }\end{array}$ & $\begin{array}{l}\text { Variación valor } \\
\text { (incertidumbre } \\
\text { reconocida) }\end{array}$ \\
\hline \multirow{2}{*}{$\begin{array}{l}\text { Convección [poco] } \\
\text { profunda ([shallow] }\end{array}$} & Ritmo de entrada de aire (entrainment rate) & 0.0003 & $0.002-0.0001$ \\
\hline & Escala de tiempo de la CAPE (CAPE time-scale) & 30 & $30-60$ \\
\hline \multirow{3}{*}{$\begin{array}{l}\text { convection) } \\
\text { Capa Límite (turbulence } \\
\text { scheme) }\end{array}$} & Longitud de mezcla (neutral mixing lenght) & 0.15 & $0.15-0.5$ \\
\hline & $\begin{array}{l}\text { Cobertura nubosa submalla (subscale cloud } \\
\text { cover w.r.t. saturation) }\end{array}$ & 0.75 & $0.5-1.0$ \\
\hline & $\begin{array}{c}\text { Factor de subcapas laminares (scaling factor of } \\
\text { laminar sublayers for scalars) }\end{array}$ & 1.0 & $0.1-50.0$ \\
\hline \multirow{4}{*}{$\begin{array}{l}\text { Nubes (large scale } \\
\text { precipitation o } \\
\text { microphysics) }\end{array}$} & $\begin{array}{c}\text { Distribución tamaño de gotas ( } \gamma \text { exponent } \\
\text { raindrop size) }\end{array}$ & 0.5 & $0.0-1.5$ \\
\hline & $\begin{array}{l}\text { Concentración gotas de nube (concentration of } \\
\text { cloud droplets) }\end{array}$ & $5 \times 10^{8}$ & $5 \times 10^{7}-1 \times 10^{9}$ \\
\hline & $\begin{array}{l}\text { Límite humedad relativa para formar nubes } \\
\text { (Critical relative humidity) }\end{array}$ & $90 \%$ & $87.5 \%-92.5 \%$ \\
\hline & Velocidad caída hielo (granizo) (Ice-fall speed) & 25.2 & $17-33$ \\
\hline \multirow{2}{*}{$\begin{array}{l}\text { Rozamiento ondas de } \\
\text { gravedad (e.g. montaña) } \\
\text { (Gravity-wave drag) }\end{array}$} & $\begin{array}{l}\text { Constante de ondas de gravedad (Gravity-wave } \\
\text { constant) }\end{array}$ & $1 \times 10^{5}$ & $7.5 \times 10^{4}-1.33 \times 10^{5}$ \\
\hline & $\begin{array}{l}\text { Número de Froude (interacción flujo con } \\
\text { montañas) }\end{array}$ & 4 & $2-4$ \\
\hline Suelo (soil-surface model) & $\begin{array}{l}\text { Índice de superficie foliar (factor of leaf area } \\
\text { index) }\end{array}$ & 1.0 & $0.5-1.5$ \\
\hline
\end{tabular}

Tabla 17.2: Ejemplo de parámetros en la física que se perturban en SPC multiparámetros. Información inspirada en dos SPC operacionales: MOGREPS del MetOffice británico [3, 9] [7, 14].

Son las incertidumbres conocidas de los valores de parámetros en la física en la Tabla 17.1 en la página 260. En los SPC que utilizan multiparámetros hay dos aproximaciones:

1. Los parámetros o números mágicos son constantes en cada uno de los miembros, pero diferentes entre los miembros.

2. Los parámetros cambian o mejor dicho evolucionan con el tiempo y/o espacio en un mismo miembro pero de manera diferente en cada miembro. Se utilizan a veces patrones de perturbación espacio-temporales (sec. 17.4.4 en la página 269 y la Figura 17.8 en la página 270).

En la Tabla 17.2 podemos ver un ejemplo tentativo de parámetros a perturbar. Destacamos dos puntos. El primer punto es que hay parametrizaciones en la física más inciertas, que se visualiza en varios parámetros con incertidumbre: convección, (microfísica de) nubes y Capa Límite Atmosférica (turbulencia). El segundo es resaltar de los valores de los parámetros:

- El gran rango de incertidumbre de la mayoría de los parámetros, por ejemplo, el ritmo de entrada del aire en la convección de 0.002 a 0.0001 ¡Un factor 20 entre ellos!

- La asimetría en la incertidumbre en alguno de ellos, por ejemplo, en el número de FROUDE en el rozamiento del flujo en las montañas, donde el modelo determinista tiene un valor 4 y en el SPC multiparámetros se tiene en cuenta la incertidumbre de 2 a 4 , pero nunca un valor mayor que 4 .

Puntos fuertes. Trata una incertidumbre real que tiene que contemplarse en el diseño de un SPC, al ser esa incertidumbre conocida y reconocida por la comunidad de modelizadores de las parametrizaciones o física. En otras palabras, tiene mucho sentido físico. Se trata de tener en cuenta las incertidumbres en el process level en inglés, es decir, al nivel del proceso dentro las parametrizaciones: donde sabemos que realmente está la incertidumbre.

Puntos débiles. A nivel práctico no funciona como se espera a nivel teórico: la realidad es que los SPC multiparámetros casi no consiguen más dispersión ni tener menos error de la media del SPC. Los resultados, en general, quedan lejos de cualquiera de los 
otros métodos. Quizás el argumento más sólido para explicar estos pobres resultados sea que, como la física en general ya es muy incierta en sí misma y, por eso, ya no tiene mucho impacto tratar la incertidumbre de unos parámetros dentro de ella (recordando el process level). Así y todo es el método que tiene más fundamento. Y hay una nueva esperanza para el process level:

perturbar estocásticamente con correlaciones espaciotemporales y dentro de cada una de las parametrizaciones, sus variables y parámetros internos [13], en vez de perturbar toda la Física como en la SPPT (ver SPC con física estocástica, sec. 17.4.4 en la página 269).

\subsubsection{SPC multifísica}

Los SPC multifísica tienen todos los miembros con la misma dinámica del modelo de predicción del tiempo (son monomodelos), pero cada uno de ellos con diferente física o diferentes combinaciones de parametrizaciones de la física.

En la Tabla 17.1 en la página 260 se ha sugerido en la última columna que la multifísica tendría en cuenta los errores o incertidumbres aleatorias de las fluctuaciones estadísticas en la física, porque, y teniendo en la cabeza la Figura 17.4 en la página 263 izquierda, utilizar diferentes físicas es como si estuviéramos seleccionando distintas salidas de los procesos submalla (líneas rojas verticales) compatibles con los procesos de malla o dinámicos.

En la Tabla 17.3 se muestra una posible combinación de diversas físicas posibles con el modelo de predicción del tiempo WRF-ARW [2, 12].

Puntos fuertes. Mejora ostensiblemente un SPC que no tiene en cuenta las incertidumbres del modelo y mejora normalmente menos que un SPC multimodelo pero bastante más que uno multiparámetros.

Puntos débiles. Es difícil asegurar la consistencia de todas las combinaciones de las parametrizaciones físicas. La física de un modelo forma un todo: las diferentes parametrizaciones tienen que estar muy bien coordinadas para tener una física consistente en el modelo. No todas las combinaciones son posibles y, además, hay parametrizaciones que no se han preparado para trabajar conjuntamente con otras. Ésto limita el número posible de combinaciones de físicaparametrizaciones y, por ende, de miembros. Por otro lado, a pesar de ser un método muy bueno para la alta resolución, muchos modelos disponen de un sólo paquete de física y no es posible aplicarlo. Finalmente, añadir también que mantener más de una física es caro, en términos de recursos humanos y, por ello, muchos centros meteorológicos desestiman esta metodología.

\begin{tabular}{|c|c|c|c|c|c|c|}
\hline \multirow{2}{*}{$\begin{array}{l}\text { Miembros / } \\
\text { Combinación } \\
\text { parametrizaciones }\end{array}$} & $\begin{array}{c}\text { Radiación } \\
\text { solar (onda } \\
\text { corta) }\end{array}$ & $\begin{array}{c}\text { Radiación } \\
\text { terrestre } \\
\text { (onda larga) }\end{array}$ & Capa Límite & Convección & $\begin{array}{c}\text { Microfísica } \\
\text { de nubes }\end{array}$ & Suelo \\
\hline 1 & Duhia & RRTM & YSU & KF & WSM5 & NOAH \\
\hline 2 & Duhia & RRTM & MYJ & Grell & WSM5 & NOAH \\
\hline 3 & Duhia & RRTM & MYJ & KF & Eta & RUC \\
\hline 4 & Duhia & CAM & YSU & BM & Lin & RUC \\
\hline 5 & Duhia & RRTM & MYJ & KF & Kessler & Thermal \\
\hline 6 & Duhia & CAM & MYJ & BM & Kessler & NOAH \\
\hline 7 & CAM & CAM & MYJ & Grell & Lin & NOAH \\
\hline 8 & CAM & RRTM & MYJ & KF & WSM6 & Thermal \\
\hline 9 & CAM & RRTM & YSU & BM & Eta & RUC \\
\hline 10 & CAM & CAM & MYJ & Grell & Thompson & RUC \\
\hline
\end{tabular}

Tabla 17.3: SPC de 10 miembros con multifísica de 10 combinaciones de diferentes esquemas de parametrizaciones de la física del modelo de predicción del tiempo WRF-ARW 3.3. 


\subsubsection{SPC multimodelo}

Los SPC multimodelo tienen un modelo diferente para cada miembro: dinámicas y físicas distintas en cada miembro. Se podría decir que el multimodelo incluye la multifísica más la incertidumbre de la parte dinámica de los modelos. Se basa en pensar que la diversidad de las configuraciones y aproximaciones en los distintos modelos desarrollados por centros meteorológicos distintos, contiene una medida válida para describir las incertidumbres, e incluso los errores, en ellos mismos [11]. ¡Casi nada! Pero ésto es más bien una justificación teórica, porque el valor real del multimodelo está en sus resultados prácticos, que son excelentes, tal como se puede ver en las verificaciones mostradas en los dos capítulos donde se describen los dos multimodelos de la Agencia Estatal de Meteorología (AEMET): el AEMET-SREPS (cap. 21 en la página 313) y AEMET- $\gamma$ SREPS (cap. 22 en la página 333).

En la Tabla 17.1 en la página 260 se ha incluido en la última columna el multimodelo como La caja negra de incertidumbres desconocidas. Ésto no es estrictamente cierto si pensamos que el multimodelo incluye a la multifísica y por ende los errores o incertidumbres aleatorias de las fluctuaciones estadísticas en la física. Pero el multimodelo es una caja negra en el sentido de que no sabemos explícitamente cómo trata las incertidumbres y los errores. Profundizando más en este argumento y, dado que sus resultados son excelentes, podemos decir que nos llevaría a adivinar que se están tratando, de alguna manera, las incertidumbres desconocidas, sobretodo, en el sentido de que no se tienen en cuenta explícitamente en ningún otro método. Por otro lado, podríamos decir que con el multimodelo los modelizadores no conocen aún suficientemente los errores y las incertidumbres de sus propios modelos $y$, por tanto, no acaban de acertar totalmente en sus técnicas para tenerlos en cuenta en sus SPC.

Se podría aducir que una de las limitaciones de los SPC multimodelo es que el número de modelos limitan el número de miembros, aunque en la práctica se combina con otras técnicas para tener en cuenta otras fuentes de incertidumbre, como las de las condiciones iniciales y, para SPC de área limitada, las de las condiciones de contorno, tal como se realiza en los AEMET-SREPS y AEMET- $\gamma$ SREPS $[5,6,8]$ (cap. 21 en la página 313 y cap. 22 en la página 333)

En la Figura 17.6 en la página siguiente se ejemplifica un SPC multimodelo puro y «de bolsillo» con sólo 4 miembros, que a la vez son 4 modelos: HARMONIEAROME (mbr001), ALARO (mbr002), WRF-ARW (mbr003) y NMMB (mbr004). Se trata de una situación de borrasca centrada en el sureste de la península ibérica con entrada de levante mediterránea desde Valencia hasta Cataluña. Es interesante observar las diferencias en la precipitación acumulada en 12 horas entre los distintos miembros/modelos: vislumbran la misma estructura general sinóptico-mesoescalar precipitante, pero con importantes desacuerdos en los detalles. Si nos fijamos en el máximo de precipitación acumulada por encima de $60 \mathrm{~mm} / 12 \mathrm{~h}$, el primer miembro (mbr001) con el modelo HARMONIEAROME lo sitúa hacia la ciudad de Tarragona, mientras el segundo (mbr002) con ALARO lo lleva más al norte en Barcelona, el WRF-ARW o tercer miembro (mbr003) a medio camino de los dos anteriores y el cuarto miembro (mbr004) en discordia, el NMMB presenta ambos máximos. La probabilidad de precipitación de más de $20 \mathrm{~mm} / 12$ horas de nuestro SPC «de bolsillo» (sólo 4 miembros) nos dibuja esa zona de la provincia de Tarragona hasta Barcelona como la de más riesgo de acumular precipitaciones mayores. La buena discriminación de la predecibilidad de los detalles del multimodelo es lo que la hace una buena técnica. Se pueden consultar también ejemplos del valor de los SPC multimodelo sobre otras metodologías en el capítulo dedicado al AEMET- $\gamma$ SREPS, en especial en el apartado (sec. 22.7.2 en la página 352) de verificación subjetiva, así como en el caso de estudio de Oliva-Gandía en la Safor de Valencia, en el apartado del AEMET- $\gamma$ SREPS repredicción (sec. 44.4 en la página 682).

Puntos fuertes. Sin duda, los resultados son excelentes. Será la mejor opción hasta que alguno de los otros métodos descritos lo supere en resultados.

Puntos débiles. Que es una caja negra para el desarrollo. Se pueden mejorar otros métodos, pero el multimodelo no se puede desarrollar directamente. No es que no se pueda mejorar un SPC multimodelo: se pueden introducir más modelos en un SPC para mejorarlo y actualizar cada modelo con versiones más nuevas y mejores que en conjunto puedan mejorar el SPC. Una de las limitaciones del multimodelo es que se necesita mantener a la vez diversos modelos en un SPC. Sea por esta razón o por razones estratégicas, los principales centros meteorológicos a nivel mundial han optado mayoritariamente por otros métodos en SPC monomodelo. 

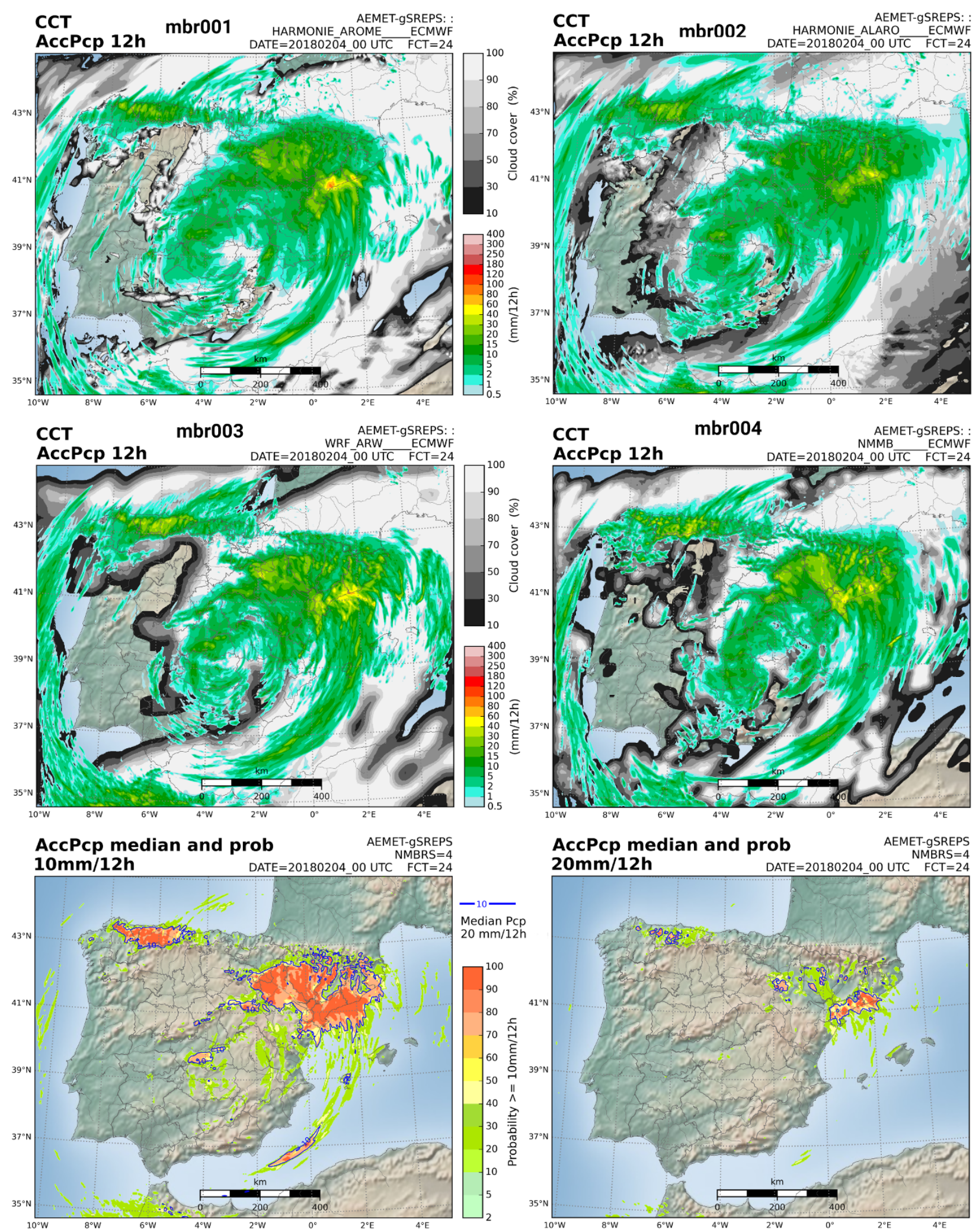

AccPcp median and prob $20 \mathrm{~mm} / 12 \mathrm{~h}$
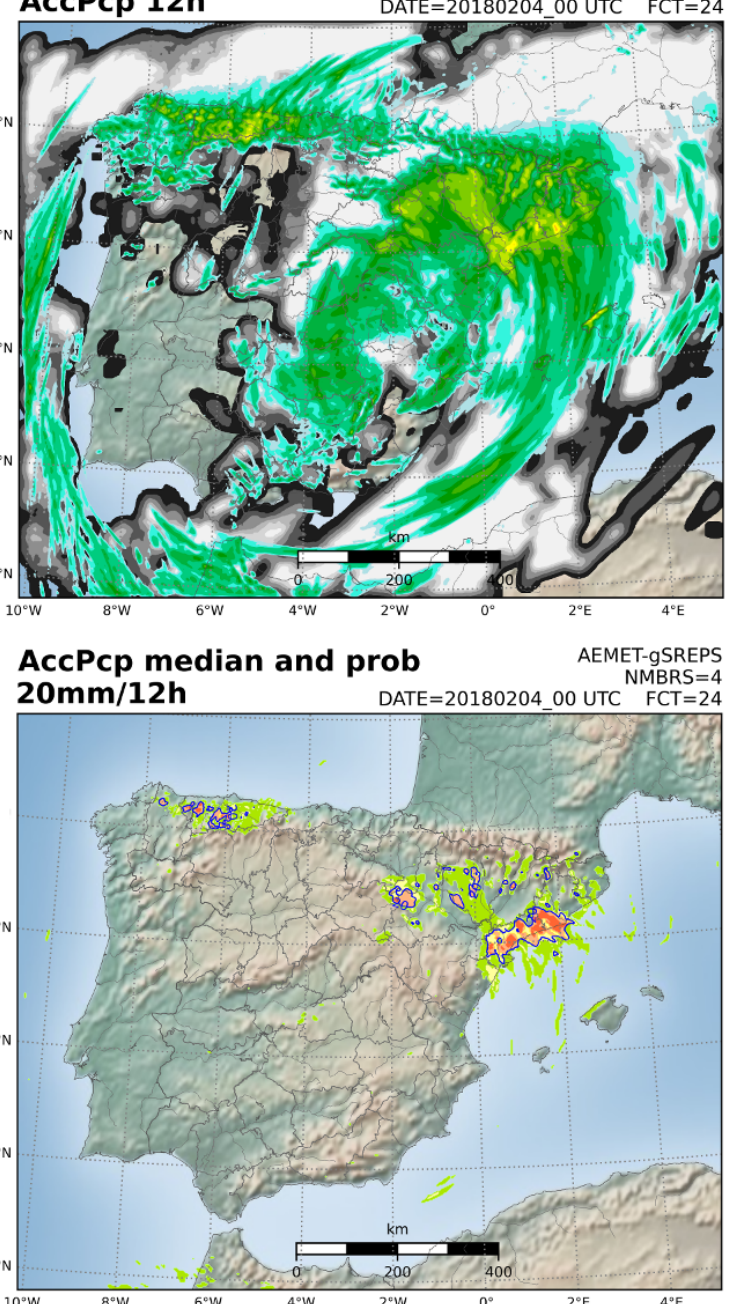

Figura 17.6: Predicción a 24 horas para el 4 de febrero de 2018 de un SPC multimodelo a 2,5 km con las condiciones de contorno del ECENS compuesto de 4 modelos: HARMONIE-AROME (mbr001), ALARO (mbrO02), WRF-ARW (mbr003) y NMMB (mbr004). En las dos primeras filas se representa la cobertura nubosa (CCT) y la precipitación acumulada en 12 horas en mm (AccPcp 12h) para los 4 miembros/modelos. En la última fila se representan las probabilidades de precipitación acumulada en 12 horas por encima de 10 (izquierda) y 20 mm (derecha), más la isolínea de la mediana de esas acumulaciones. 


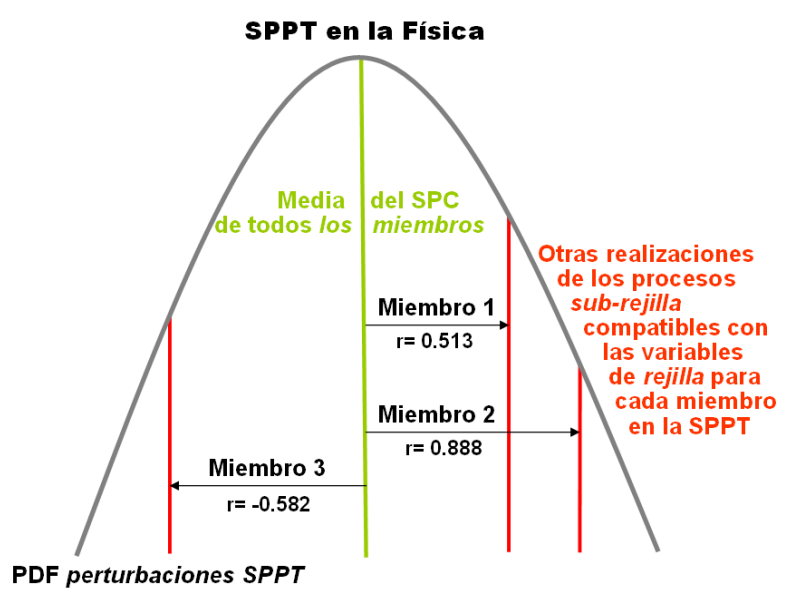

Figura 17.7: Transcripción de la Figura 17.4 en la página 263 al aplicarse SPPT: cada miembro del SPC perturba la salida de la física con un número aleatorio distinto tomando posibles salidas distintas pero igualmente plausibles.

\subsubsection{SPC con física estocástica (parame- trizaciones estocásticas)}

Los SPC con parametrizaciones estocásticas tratan los errores/incertidumbres aleatorias de la Tabla 17.1 en la página 260 perturbando o modificando, con números aleatorios (al azar), variables de los modelos pero de diferente manera en cada miembro de un SPC. Y, en cierto sentido, ya se empieza a cumplir la predicción de la cita del célebre físico y meteorólogo EDWARD N. LORENZ: «Creo que en última instancia los modelos ... serán estocásticos, es decir, aparecerán números aleatorios en algún lugar de las derivadas temporales». En la Tabla 17.1 en la página 260 podemos observar que hay referenciadas dos parametrizaciones estocásticas que son las dos más ampliamente utilizadas en los SPC globales y regionales (ver Tabla 17.5 en la página 272):

SPPT o perturbaciones estocásticas de las tendencias de la física $[4,13,16]$, en los artículos científicos en inglés Stochastic Peturbations of Parameterization Tendencies, que se supone que tienen en cuenta las fluctuaciones estadísticas en la física de la Figura 17.4 en la página 263 izquierda al igual que el método de multifísica

SKEB o retrodispersión estocástica de energía cinética $[1,20]$, en inglés Stochastic Kinetic Energy Backscatter, que reproduce una transferencia de energía cinética a mayores escalas (Tabla 17.1 en la página 260) desde escalas pequeñas de submalla donde se pierde energía en los modelos, debido que se observa que parte de ella en la atmósfera real vuelve a las estructuras mesoescalares y sinópticas.

Ambas parametrizaciones estocásticas tienen diversas variantes en su utilización operativa, e incluso pueden aplicarse combinadamente como podemos ver en la Tabla 17.5 en la página 272 en el caso del SPC global ECENS del ECMWF. Se pueden combinar debido a que tratan incertidumbres en los modelos diferentes como ya se ha contado (ver otra vez Tabla 17.1 en la página 260).

Vamos a desarrollar aquí sólo la SPPT por su relevancia en su uso operativo y porque es más fácil de entender que la SKEB. La SPPT se fundamenta en perturbar («multiplicar») la salida de la física con un número aleatorio diferente en cada miembro del SPC. De esta manera, cada miembro del SPC puede recoger una de las salidas posibles de la física o procesos submalla igualmente plausibles con los procesos de malla de la Figura 17.4 en la página 263 izquierda, tal como mostramos en la Figura 17.7.

Stochastic Peturbations of Parameterization Tendencies (SPPT) $[1,4,16,20])$. Matemáticamente la SPPT se fundamenta en multiplicar por números aleatorios (conocido como ruido multiplicativo, multiplicative noise en inglés) las tendencias de las salidas de las parametrizaciones, es decir, añadir el término extra $\delta P_{X}$ en la ecuación de pronóstico del modelo:

$$
\frac{\partial X}{\partial t}=D_{X}+K_{X}+P_{X}+\delta P_{X}
$$

con:

$$
\delta P_{X}=\alpha r P_{X}
$$

donde $X$ es una variable del modelo (temperatura $T$, humedad específica $q$ y componentes del viento $U$ y $V), \frac{\partial}{\partial t}$ es la variación en el tiempo, $D_{X}$ es la tendencia de la dinámica, $K_{X}$ la tendencia de la difusión, $P_{X}$ la tendencia de la física, $\alpha$ es el reajuste de SPPT en la vertical (Figura 17.8 en la página siguiente derecha) y $r$ es el número aleatorio. La tendencia de la técnica SPPT es el término $\delta P_{X}$ que multiplica la tendencia de las variables de la física por el valor $r$, reajustado en la vertical por el parámetro $\alpha$, modificando efectivamente el valor del término de la tendencia de la física $P_{X}$ en la ecuación. El reajuste $\alpha$ (tapering en inglés) al tomar valores 0 en la estratosfera y en la PBL (ver Figura 17.8 en la página siguiente Derecha) no las perturba, pero la SPPT sí que perturba sin restricciones en los niveles medios troposféricos donde $\alpha=1$. 
Stochastic Kinetic Energy Backscatter (SKEB [1, 20]). Soluciona un defecto de los modelos. Una fracción de la energía cinética disipada por el modelo cerca de la resolución de la malla es transferida a las escalas donde se resuelve el flujo a través de una función de forzamiento del flujo. La formulación matemática viene dada por:

$$
F_{\Psi}(\lambda, \mu, v, t)=\sqrt{b_{R} D_{t o t}} F(\lambda, \mu, v, t)
$$

con:

$$
D_{t o t}=D_{\text {num }}+D_{G W D}+D_{c} \sin ^{2} \phi
$$

donde $F_{\Psi}$ es la función de forzamiento del flujo y donde se suponen tres fuentes de la energía disipada: por producción insuficiente en convección profunda $\left(D_{C}\right)$, por rozamiento orográfico y ondas de gravedad $\left(D_{G W D}\right)$, y por disipación/difusión numérica $\left(D_{\text {num }}\right)$.

Un punto fundamental en el diseño de las parametrizaciones estocásticas en general y, de la SPPT en concreto, es que los números aleatorios se aplican con correlaciones espacio-temporales. ¿Qué es esto de las correlaciones? Pues que entre puntos de malla o de grid vecinos se cogen números aleatorios parecidos (correlación espacial) durante un tiempo largo en la integración del modelo (correlación temporal): son los patrones de perturbación. Asentemos ideas con el ejemplo de correlación espacial mostrado en la Figura 17.8 izquierda: se representan en cajitas grandes de unos 180 x $180 \mathrm{~km}$ los números aleatorios aplicados durante 3 horas: la correlación temporal. Después de esas 3 horas cambian todos los valores de los números de todas las cajitas. Anotamos que los patrones de perturbación actuales son suaves sin saltos espaciales ni temporales bruscos como los mostrados. Pero el patrón en cajitas mostrado tiene una vertiente sentimental e histórica, porque fue como se hizo en su primera implementación por ROBERTO BUIZZA allá por 1999 [4].

Desde las primeras implementaciones surgieron algunos problemas y dudas teóricas sobre perturbar de este modo la parte de la física de los modelos. Teóricamente no había tanta incertidumbre en la estratosfera en los niveles altos y, perturbar la turbulencia, ya muy incierta en sí misma, en los niveles bajos daba problemas. Así, normalmente, se aplica un ajuste a la SPPT representada en la Figura 17.8 derecha. Los valores igual a 1 de la troposfera media muestran dónde se aplica la SPPT y los valores 0 en la PBL y la troposfera, dónde no se usa.

Finalmente, debemos resaltar que hay una gran diversidad de maneras diferentes de aplicar la SPPT, perturbando, no toda la física como en la implementación básica, sino las parametrizaciones individuales dentro de ella, de manera más o menos independiente; y/o perturbando también de manera diferente cada variable del modelo, etcétera. Aunque la aplicación más básica y común es perturbar toda la física y todas las variables del modelo por igual.
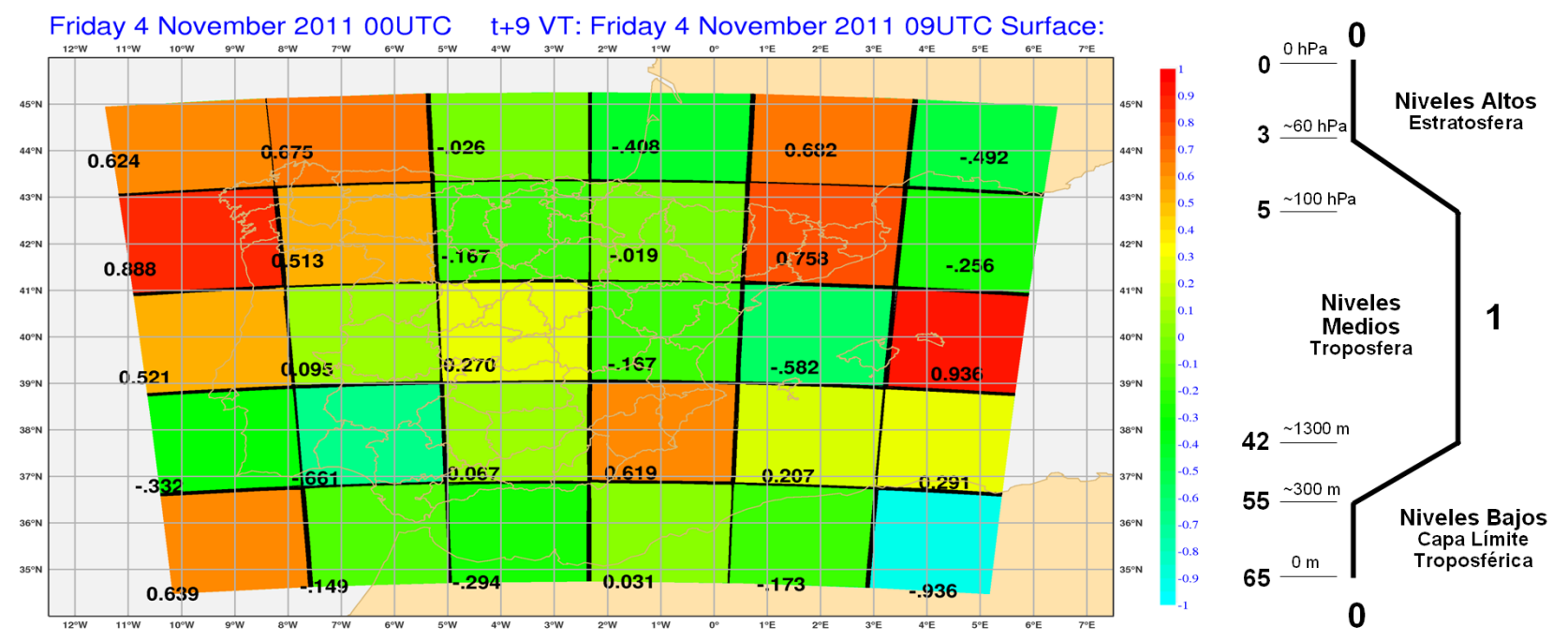

Figura 17.8: Izquierda: correlaciones espaciales horizontales en cajas o cajitas rectangulares abarcadas por los números aleatorios en la SPPT. En negro se plotean sus valores aleatorios entre -1 y 1. Derecha: reajuste (tapering en inglés) vertical de los números aleatorios en la SPPT para no perturbar en la PBL y en la estratosfera. 


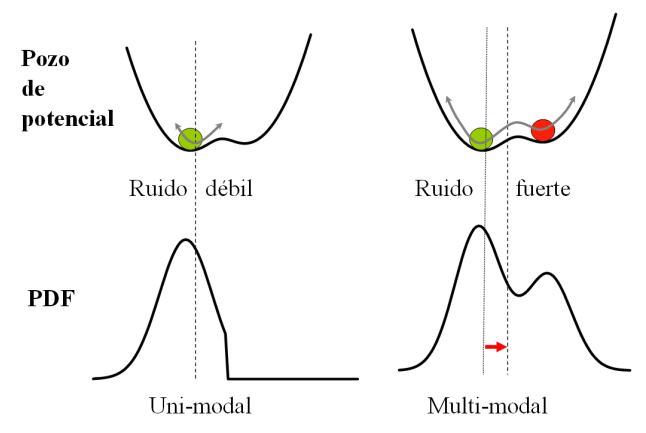

Figura 17.9: Justificación de la validez conceptual de las parametrizaciones estocásticas (PE) en SPC monomodelo: capacidad de las PE en aumentar la dispersión (variabilidad) con un ruido fuerte y a la vez modificar la PDF mejorando la media del SPC (flecha roja) (siguiendo las ideas y figuras originales de J. BERNER y G. SHUTTS).

Puntos fuertes SPPT. Ostenta los mejores resultados con SPC monomodelo. Simple de implementar, al menos respecto a otras parametrizaciones estocásticas. Conceptualmente trata una incertidumbre reconocida por los meteorólogos-modelizadores: las fluctuaciones estadísticas de la física. Se puede combinar con otras técnicas e incluso parametrizaciones estocásticas como la SKEB. En la Figura 17.9 se representa uno de los argumentos aducidos para explicar los buenos resultados de las parametrizaciones estocásticas: un aumento de la dispersión que (sorprendentemente) a la vez disminuye el error medio del SPC, mejorando la media del SPC. Teóricamente, se razona que en un
SPC, al aumentar la perturbación o ruido (de ruido débil a fuerte), se aumenta la variabilidad interna del modelo de predicción del tiempo, acercándolo a la variabilidad natural de la atmósfera real y, de este modo, el modelo puede explorar (bolita roja) una parte (del pozo de potencial) inaccesible antes para el mismo.

Puntos débiles SPPT. Se precisa de un gran número de experimentos para su ajuste o tuneo, cada vez que se hacen cambios en el modelo del SPC, especialmente en la parte de la física. Limitada capacidad de evolución.

\subsection{Discusión final}

Valoración subjetiva de las metodologías del error del modelo. En la Tabla 17.4, resumimos subjetivamente los puntos fuertes y débiles a nivel más bien práctico de los distintos métodos para tener en cuenta las incertidumbres y los errores en los SPC, pero haciendo hincapié en la diferencia en su uso común en incertidumbres sinópticas y meso- $\alpha(200-2000 \mathrm{~km})$ respecto a escalas espacio-temporales más pequeñas como la meso- $\beta$ y meso- $\gamma(2-200 \mathrm{~km})$ (ver sec. 22.1 en la página 334). La Tabla, basada en multitud de SPC regionales, pretende ser una guía útil de cómo tratar los errores y las incertidumbres a la hora de diseñar un SPC.

\begin{tabular}{|c|c|c|c|c|c|}
\hline $\begin{array}{c}\text { Métodos errores e } \\
\text { incertidumbres } \\
\text { SPC }\end{array}$ & $\begin{array}{c}\text { meso- } \beta \text { y } \\
\text { meso- } \gamma \\
0-12 \text { horas }\end{array}$ & $\begin{array}{c}\text { Sinópticas y } \\
\text { meso- } \alpha \\
\text { Más de 12 horas }\end{array}$ & Ventajas & Desventajas & $\begin{array}{c}\text { Puntuación } \\
\text { final }\end{array}$ \\
\hline \hline multiparámetros 5 & +1 & -1 & $\begin{array}{c}\text { Un modelo } \\
+2\end{array}$ & $\begin{array}{c}\text { Precisa de buen } \\
\text { conocimiento de las } \\
\text { parametrizaciones -1 }\end{array}$ & $6(7)$ \\
\hline multifísica 5 & +2 & 0 & $\begin{array}{c}\text { Un modelo } \\
+2\end{array}$ & $\begin{array}{c}\text { Consistencia y } \\
\text { disponibilidad de } \\
\text { diferentes físicas -1 }\end{array}$ & $8(8)$ \\
\hline $\begin{array}{c}\text { física estocástica } \\
\text { (parametrizacio- } \\
\text { nes): SPPT } \\
5\end{array}$ & $i 0 ?$ & +2 & $\begin{array}{c}\text { Simple } \\
\text { parametrización } \\
+3\end{array}$ & $\begin{array}{c}\text { Dificultad de tuneo o } \\
\text { ajuste con multitud de } \\
\text { experimentos -1 }\end{array}$ & $9(7)$ \\
\hline multimodelo 5 & +2 & +2 & $\begin{array}{c}\text { Simple Tuneo no } \\
\text { necesario +2 }\end{array}$ & $\begin{array}{c}\text { Mantener un buen } \\
\text { número de modelos }-1\end{array}$ & $10(8)$ \\
\hline
\end{tabular}

Tabla 17.4: Puntuación subjetiva de los diferentes métodos para tener en cuenta los errores e incertidumbres en los SPC regionales, haciendo hincapié en su valor en dos espacios-temporales, el de los fenómenos atmosféricos a escalas mesoescalares (2-200 km, hasta 12 horas) y el de las escalas sinópticas (200-2000 km, más de 12 horas), según la clasificación de Orlanski [15]. Entre paréntesis en la puntuación final está la puntuación sin considerar la escala sinóptica. 
Como ejemplo, contaremos que la misma Tabla 17.4 en la página anterior fue la utilizada en su día en el diseño del futuro AEMET- $\gamma$ SREPS (ver cap. 22 en la página 333). Se escogió un SPC multimodelo por tener la puntuación más alta. A cambio, la desventaja (no despreciable) fue, y es, instalar y mantener 4 modelos de predicción del tiempo.

Metodologías del error del modelo en SPC operativos. En la Tabla 17.5 se muestran los distintos métodos que diversos servicios meteorológicos a nivel internacional han escogido para tener en cuenta los errores e incertidumbres de sus modelos en sus SPC globales y regionales operativos. Subrayaremos dos aspectos fácilmente observables en la tabla.

El primer aspecto es que sobresalen los métodos de la multifísica (5 entradas) y la física estocástica (6 entradas) como los más usados, y el multiparámetros (3 entradas) y el multimodelo ( 2 entradas) como los menos usados. El multiparámetros muy probablemente es poco usado debido a sus limitados (¡Pobres!) resultados, y en realidad es sólo usado como único método para considerar el error del modelo en un SPC regional de alta resolución, el COSMO-DE [7, 19], que es donde parece tener algo de habilidad (destreza, pericia, skill, ver sec. 15.2.4 en la página 212) en las primeras horas de predicción como se ha consignado en la Tabla 17.4 en la página anterior. Para el multimodelo se explica su bajo uso, no por sus resultados, que son excelentes, sino por dos razones bien distintas: por la dificultad en mantener varios modelos a la vez, y por la política de muchos Servicios Meteorológicos de utilizar sólo su propio y único modelo: ¡Política estratégica monomodelo!

El segundo aspecto es que casi la mitad de los SPC apuestan por una estrategia combinada, es decir, utilizar dos metodologías a la vez. Ya hemos comentado y resaltado de la Tabla 17.1 en la página 260, que el hecho de que diferentes metodologías del error del modelo sólo tengan en cuenta ciertos aspectos de la clasificación de errores e incertidumbres, justifica a nivel teórico, y en la práctica también, que se puedan combinar sin problemas. Por ejemplo, el ECENS del ECMWF utiliza dos parametrizaciones estocásticas a la vez, SPPT y SKEB; y el CMC en su GEPS, adicionalmente a la SPPT y la SKEB, las combina con la metodología de multifísica ¡Tres métodos a la vez!

\begin{tabular}{|c|c|c|c|c|}
\hline \multirow{2}{*}{$\begin{array}{l}\text { SPC (globales o regionales) } \\
\text { [Servicio Meteorológico] }\end{array}$} & \multicolumn{4}{|c|}{ Metodologías de SPC en el error del modelo } \\
\hline & multiparámetros 3 & multifísica 5 & multimodelo 2 & $\begin{array}{c}\text { física estocástica } \\
6\end{array}$ \\
\hline ECENS (G) [ECMWF] & & & & SPPT + SKEB \\
\hline $\begin{array}{c}\text { AEMET- } \gamma \text { SREPS }(\mathrm{R}) \\
{[\mathrm{AEMET}]}\end{array}$ & & & $\begin{array}{c}\text { AROME + } \\
\mathrm{ALARO} \mathrm{+} \mathrm{WRF} \mathrm{+} \\
\text { NMMB }\end{array}$ & \\
\hline PEARP (G) [MétéoFrance] & & Multiphysics & & \\
\hline $\begin{array}{c}\text { AROME-EPS (R) } \\
\text { [MétéoFrance] }\end{array}$ & & & & SPPT \\
\hline $\begin{array}{l}\text { MOGREPS-G }(\mathrm{G}) \\
\text { [MetOffice] }\end{array}$ & $\begin{array}{l}\text { RP (Random } \\
\text { Parameters) }\end{array}$ & & & SKEB \\
\hline COSMO-DE (R) [DWD] & Multiparameter & & & \\
\hline $\begin{array}{l}\text { COSMO-LEPS (R) } \\
\text { [ARPA-SIM] }\end{array}$ & Multiparameter & Multiphysics & & \\
\hline GEFS (G) [NOAA-NCEP] & & & & SPPT \\
\hline SREF (R) [NOOA-NCEP] & & $\begin{array}{l}\text { Multiphysics } \\
(\mathrm{NAM}+\mathrm{GFS}+ \\
\text { NCAR+RAP) }\end{array}$ & $\begin{array}{c}\text { WRF-ARW + } \\
\text { WRF-NMM + } \\
\text { NMMB }\end{array}$ & \\
\hline GEPS (G) [JMA] & & Multiphysics & & \\
\hline GEPS (G) [CMC] & & Multiphysics & & SPPT + SKEB \\
\hline REPS (R) [CMC] & & & & SPPT \\
\hline
\end{tabular}

Tabla 17.5: Resumen (no exhaustivo) de los esquemas de las distintas metodologías de diversos SPC globales y regionales operativos para tener en cuenta los errores e incertidumbres de los modelos. 

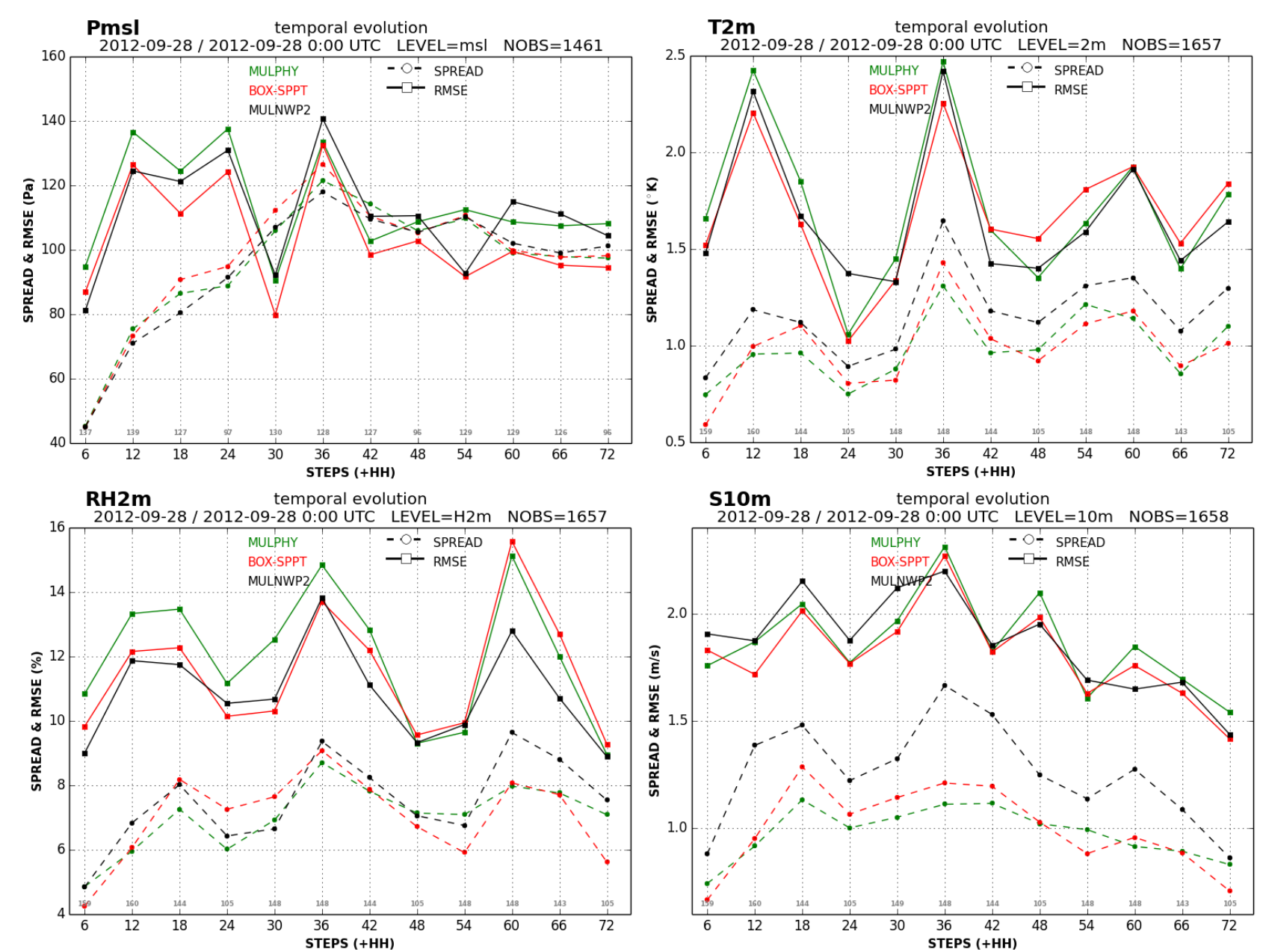

Figura 17.10: Diagramas de evolución con el alcance predictivo de la dispersión y el error de la presión reducida a nivel de mar (Pmsl), temperatura y humedad relativa de garita a dos metros $(T 2 m)$ y viento de anemómetro a 10 metros $(S 10 m)$ comparando tres metodologías para tener en cuenta los errores y las incertidumbres del modelo en 3 SPC con las mismas condiciones de contorno del ECENS: multifísica (verde), física-estocástica SPPT (rojo) y multimodelo (negro).

Verificaciones objetivas de 3 metodologías en SPC para el error del modelo. En la Figura 17.10 se muestran dos verificaciones objetivas en la península ibérica cada 6 horas hasta 3 días ( 72 horas) con los diagramas de evolución con el alcance predictivo de dispersión y error e histogramas de rango respectivamente (sec. 15.8 en la página 222), de 4 variables meteorológicas, la presión reducida a nivel de mar (Pmsl), temperatura y humedad relativa de garita a dos metros (T2m y RH2m) y viento a la altura del anemómetro a 10 metros (S10m). Se comparan tres SPC puros, con el modelo HARMONIE-AROME y condiciones de contorno del ECENS (ECMWF), utilizando cada uno una metodología distinta para tener en cuenta error e incertidumbres del modelo:

1. Multifísica (en verde) con dos físicas: AROME y ALARO

2. Física estocástica (en rojo) con la parametriza- ción estocástica SPPT

3. Multimodelo (en negro) con dos modelos: HARMONIE-AROME y NMMB.

El resumen de la interpretación es fácil: el multimodelo es la metodología que mejor funciona, mientras que multifísica y física estocástica SPPT no funcionan mal y sus resultados son similares.

Más específicamente, en la Figura 17.10 con el diagrama de evolución con el alcance predictivo de la dispersión y el error vemos, por ejemplo, con la temperatura a dos metros (T2m), que el multimodelo (en negro) tiene la mejor habilidad (skill), porque las curvas de error (RMSE en negro continuo) y de dispersión (línea negra discontinua) están más cerca entre ellas, siendo muchas veces el error menor y siempre la dispersión mayor que en la multifísica (verde) y la SPPT de la física estocástica (rojo). 
En la Figura 17.11, con los histogramas de rango y fijándonos también en la T2m (sugerimos analizar las otras tres variables: Pmsl, RH2m y S10m), vemos que la consistencia del SPC multimodelo es claramente superior al multifísica y a la SPPT de física estocástica, al tener un diagrama bastante más plano que los otros dos SPC, aunque aún adolece de cierta falta de dispersión al ser aún visible una cierta forma en U (sec. 15.8.1 en la página 222).

Para finalizar el capítulo, mencionaremos que en los
SPC actuales se están utilizando metodologías específicas para tener en cuenta las incertidumbres en la superficie terrestre y marítima con sus interacciones con la atmósfera: las perturbaciones de la superficie, es decir, en la condición de contorno inferior de los modelos. Dependiendo de cada metodología y cómo se aplique, cae más del lado de las incertidumbres en las condiciones de contorno o más en el campo del error del modelo. Y las perturbaciones de la superficie van, e irán en un futuro, cobrando más interés en los SPC cada vez con una resolución horizontal más alta.
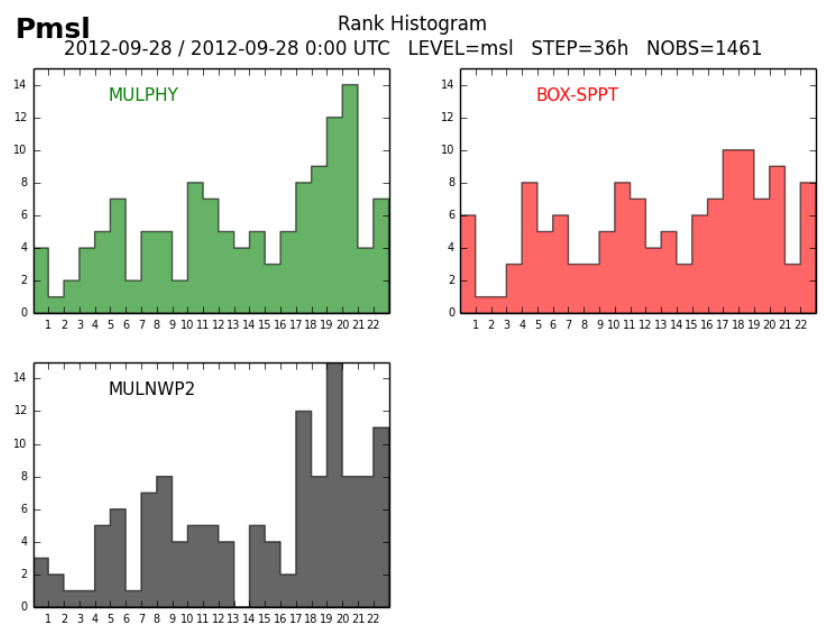

RH2 m Rank Histogram
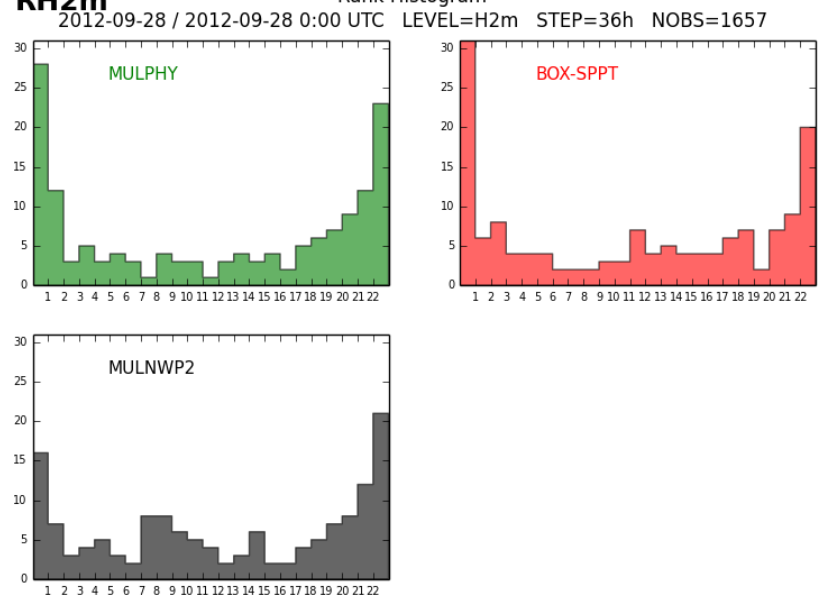

T2 2012-09-28 / 2012-09-28 0:00 Rank Histogram
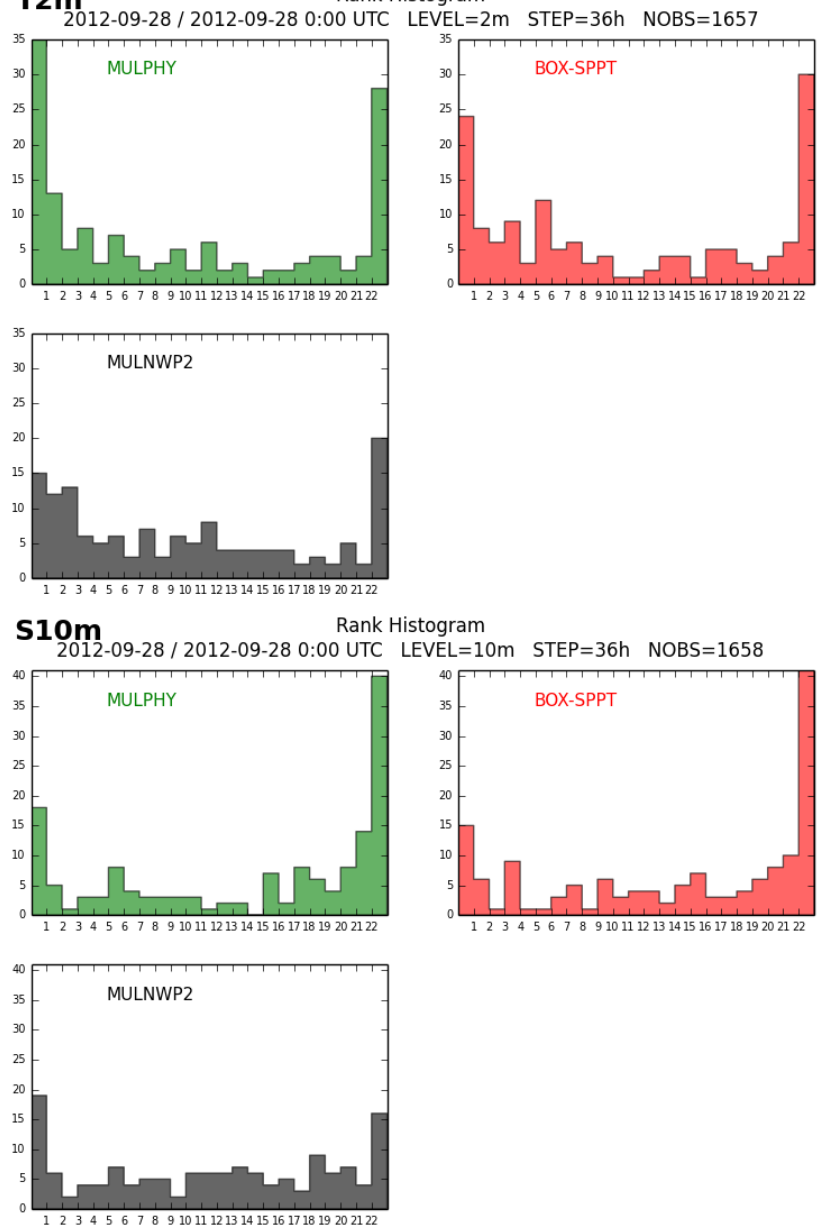

Figura 17.11: Igual que la Figura 17.10 en la página anterior, pero con los histogramas de rango. 


\subsection{Referencias}

[1] Berner, J. y col. "A Spectral Stochastic Kinetic Energy Backscatter Scheme and Its Impact on Flow-Dependent Predictability in the ECMWF Ensemble Prediction System". En: Journal of the Atmospheric Sciences 66.3 (mar. de 2009), páginas 603-626. ISSN: 00224928. DOI: $10.1175 / 2008 J A S 2677.1$ (citado en páginas 269,270 ).

[2] Berner, J y col. "Model Uncertainty in a Mesoscale Ensemble Prediction System: Stochastic versus Multiphysics Representations”. En: Monthly Weather Review 139.6 (2011), páginas 1972-1995. DOI: 10.1175/ 2010MWR3595. 1 (citado en página 266).

[3] Bowler, Neill E. y col. "The MOGREPS short-range ensemble prediction system". En: Quarterly Journal of the Royal Meteorological Society 134.632 (abr. de 2008), páginas 703-722. ISSN: 00359009. DOI: 10 . 1002/qj . 234 (citado en página 265).

[4] Buizza, Roberto, Miller, Martin J y PALMER, Tim N. "Stochastic representation of model uncertainties in the ECMWF ensemble prediction system". En: Quarterly Journal of the Royal Meteorological Society 125.560 (ago. de 1999), páginas 2887-2908. ISSN: 00359009. DOI: 10 . $1002 / \mathrm{qj}$. 49712556006 (citado en páginas 269, 270).

[5] Callado, Alfons y col. "Ensemble Forecasting". En: Climate Change and Regional/Local Responses. Editado por RAY, Pallav. InTech, mayo de 2013. ISBN: 978-95351-1132-0. DOI: 10 . 5772/55699 (citado en página 267 ).

[6] García-Moya, José Antonio y col. "Predictability of short-range forecasting: A multimodel approach". En: Tellus, Series A: Dynamic Meteorology and Oceanography 63.3 (mayo de 2011), páginas 550-563. ISSN: 02806495. DOI: $10.1111 / \mathrm{j} .1600-0870$. 2010.00506.x (citado en página 267).

[7] Gebhardt, C. y col. "Uncertainties in COSMO-DE precipitation forecasts introduced by model perturbations and varia- tion of lateral boundaries". En: Atmospheric Research 100.2-3 (mayo de 2011), páginas 168-177. ISSN: 0169-8095. DOI: 10 . 1016/J . ATMOSRES . 2010 . 12.008 (citado en páginas 265, 272).

[8] Hagedorn, Renate y col. "Comparing TIGGE multimodel forecasts with reforecastcalibrated ECMWF ensemble forecasts". En: Quarterly Journal of the Royal Meteorological Society 138.668 (oct. de 2012), páginas 1814-1827. ISSN: 00359009. DOI: 10 . 1002/qj . 1895 (citado en página 267).

[9] Hagelin, Susanna y col. "The Met Office convective-scale ensemble, MOGREPSUK”. En: Quarterly Journal of the Royal Meteorological Society 143.708 (2017), páginas 2846-2861. DOI: 10 . 1002/qj . 3135 (citado en página 265).

[10] Hall, David M y col. "Dynamical Core Model Intercomparison Project (DCMIP) tracer transport test results for CAM-SE". En: Quarterly Journal of the Royal Meteorological Society 142.697 (2016), páginas 1672-1684. ISSN: 1477-870X. DOI: 10 . 1002/qj . 2761 (citado en página 262).

[11] Hou, Dingchen, KalnaY, Eugenia y DroeGEMEIER, Kelvin K. "Objective verification of the SAMEX'98 ensemble forecasts". En: Monthly Weather Review 129.1 (2001), páginas 73-91 (citado en páginas 262, 267).

[12] JANKOV, Isidora y col. "A Performance Comparison between Multiphysics and Stochastic Approaches within a North American RAP Ensemble". En: Monthly Weather Review 145.4 (2017), páginas 1161-1179. DOI: 10. 1175/MWR-D-16-0160 . 1 (citado en página 266).

[13] Leutbecher, Martin y col. "Stochastic representations of model uncertainties at ECMWF: state of the art and future vision". En: Quarterly Journal of the Royal Meteorological Society 143.707 (2017), páginas 2315-2339. ISSN: 1477-870X. DOI: 10 . 1002/qj . 3094 (citado en páginas 262, 266, 269). 
[14] McCABE, Anne y col. "Representing model uncertainty in the Met Office convectionpermitting ensemble prediction system and its impact on fog forecasting". En: Quarterly Journal of the Royal Meteorological Society 142.700 (2016), páginas 2897-2910. ISSN: 1477-870X. DOI: 10 . 1002/qj . 2876 (citado en página 265).

[15] ORlanski, L. "A rational subdivision of scale for atmospheric processes". En: Bull. Amer. Meteor. Soc. 56 (1975), páginas 527-530 (citado en página 271).

[16] Palmer, T N y col. "Stochastic parametrization and model uncertainty". En: ECMWF Tech. Memo 598 (2009), páginas 1-42 (citado en página 269).

[17] PALMer, Tim N. "On parametrizing scales that are only somewhat smaller than the smallest resolved scales, with application to convection and orography". En: Proceedings of the ECMWF workshop on New insights and approaches to convective parametrization, ECMWF, Shinfield Park, Reading RG2-9AX, UK. 1997, páginas 328-337 (citado en página 262).

[18] Palmer, Tim N. y col. "Development of a European multimodel ensemble system for seasonal-to-interannual prediction (DEMETER)". En: Bulletin of the American Meteorological Society 85.6 (2004), páginas 853-872 (citado en página 262).
[19] Peralta, C y col. "Accounting for initial condition uncertainties in COSMO-DEEPS". En: Journal of Geophysical Research: Atmospheres 117.D7 (2012), n/a-n/a. ISSN: 2156-2202. DOI: 10.1029/2011JD016581 (citado en página 272).

[20] SHUTTs, Glenn. "A kinetic energy backscatter algorithm for use in ensemble prediction systems". En: Quarterly Journal of the Royal Meteorological Society 131.612 (2005), páginas 3079-3102. DOI: 10.1256/qj .04.106 (citado en páginas 269, 270).

[21] ShutTs, Glenn y Callado Pallarès, Alfons. "Assessing parametrization uncertainty associated with horizontal resolution in numerical weather prediction models". En: Philosophical transactions. Series A, Mathematical, physical, and engineering sciences 372 (2014) (citado en páginas 262, 263).

[22] ShutTs, G y col. "Representing model uncertainty: Stochastic parametrizations at ECMWF". En: ECMWF Newsletter 129 (2011), páginas 19-24 (citado en página 260).

[23] VVAA. Dynamical Core Model Intercomparison Project (DCMIP). URL: https : / / earthsystemcog . org / projects / dcmip-2016/ (citado en página 262). 